\title{
OPTIMAL QUADRATURE FORMULAS IN THE SENSE OF SARD IN $W_{2}^{(m, m-1)}$ SPACE
}

\author{
KH.M.SHADIMETOV, A.R.HAYOTOV
}

\begin{abstract}
In this paper in $W_{2}^{(m, m-1)}(0,1)$ space the problem of construction of optimal quadrature formula in the sense of Sard is considered and using S.L.Sobolev's method it is obtained new optimal quadrature formula of such type. For the optimal coefficients explicit formulas are obtained. Furthermore, the numerical results which confirm the theoretical results of this work is given.

MSC: $65 \mathrm{D} 32$.

Keywords: optimal quadrature formulas; the error functional; the extremal function; Hilbert space; optimal coefficients.
\end{abstract}

\section{Introduction. Statement of the problem}

We consider the following quadrature formula

$$
\int_{0}^{1} \varphi(x) d x \cong \sum_{\beta=0}^{N} C_{\beta} \varphi\left(x_{\beta}\right)
$$

with the error functional

$$
\ell(x)=\varepsilon_{[0,1]}(x)-\sum_{\beta=0}^{N} C_{\beta} \delta\left(x-x_{\beta}\right),
$$

where $C_{\beta}$ are the coefficients and $x_{\beta}$ are the nodes of formula $(1.1), x_{\beta} \in[0,1], \varepsilon_{[0,1]}(x)$ is the indicator of the interval $[0,1], \delta(x)$ is Dirac's delta-function, function $\varphi(x)$ belongs to Hilbert space $W_{2}^{(m, m-1)}(0,1)$. The norm of functions in this space is defined by the following equality

$$
\left\|\varphi(x) \mid W_{2}^{(m, m-1)}(0,1)\right\|=\left\{\int_{0}^{1}\left(\varphi^{(m)}(x)+\varphi^{(m-1)}(x)\right)^{2} d x\right\}^{1 / 2} .
$$

The difference

$$
(\ell(x), \varphi(x))=\int_{0}^{1} \varphi(x) d x-\sum_{\beta=0}^{N} C_{\beta} \varphi\left(x_{\beta}\right)=\int_{-\infty}^{\infty} \ell(x) \varphi(x) d x
$$

is called the error of the quadrature formula (1.1). The error of the formula (1.1) is linear functional in $W_{2}^{(m, m-1) *}(0,1)$, where $W_{2}^{(m, m-1) *}(0,1)$ is the conjugate space to the space $W_{2}^{(m, m-1)}(0,1)$.

By Cauchy-Schwartz inequality

$$
|(\ell(x), \varphi(x))| \leq\left\|\varphi(x)\left|W_{2}^{(m, m-1)}(0,1)\|\cdot\| \ell(x)\right| W_{2}^{(m, m-1) *}(0,1)\right\|
$$


the error (1.4) of formula (1.1) is estimated with the help of the norm

$$
\left\|\ell(x)\left|W_{2}^{(m, m-1) *}(0,1) \|=\sup _{\left\|\varphi(x) \mid W_{2}^{(m, m-1)}(0,1)\right\|=1}\right|(\ell(x), \varphi(x)) \mid\right.
$$

of the error functional (1.2). Consequently, estimation of the error of the quadrature formula (1.1) on functions of the space $W_{2}^{(m, m-1)}(0,1)$ is reduced to finding the norm of the error functional $\ell(x)$ in the conjugate space $W_{2}^{(m, m-1) *}(0,1)$.

Obviously the norm of the error functional $\ell(x)$ depends on the coefficients $C_{\beta}$ and the nodes $x_{\beta}$. The problem of finding the minimum of the norm of the error functional $\ell(x)$ by coefficients $C_{\beta}$ and by nodes $x_{\beta}$, is called the S.M.Nikolskii problem, and obtained formula is called optimal quadrature formula in the sense of Nikolskii. This problem was first considered by S.M.Nikolskii [15]. The problem were further investigated by many authors for various cases (see e.g. $[2-5,16,33]$ and references therein). Minimization of the norm of the error functional $\ell(x)$ by coefficients $C_{\beta}$ when the nodes are fixed is called Sard's problem. And the obtained formula is called optimal quadrature formula in the sense of Sard. This problem was first investigated by A.Sard [17].

There are several methods for the construction of optimal quadrature formulas in the sense of Sard such as the spline method, the $\varphi(x)$ - function method (see e.g. [2], [24]) and Sobolev's method. Note the Sobolev method is based on the construction of a discrete analogue of a linear differential operator (see e.g. [27-29]). In different spaces based on these methods, the Sard problem was investigated by many authors (see, for example, [1,2,4,6-8,11-14,18-20,23-29,31,32] and references therein).

Furthermore, explicit formulas for coefficients of optimal quadrature formulas for any $m$ and for any number $N+1$ of the nodes $x_{\beta}$ in the space $L_{2}^{(m)}$ when the nodes are equally spaced were obtained in the works $[11,18,19]$. Here $L_{2}^{(m)}$ is the Sobolev space of functions, which $m$-th generalized derivative is square integrable. In the work [20] positiveness of the coefficients of optimal quadrature formulas in $L_{2}^{(m)}(0,1)$ space is investigated.

It should be noted that in the works $[8,12]$, using the $\varphi$ - function method, investigated the problem of construction of optimal quadrature formulas in the sense of Sard which are exact for solutions of linear differential equations and several examples given for some number of the nodes.

The main aim of the present paper is to solve the Sard problem in the space $W_{2}^{(m, m-1)}(0,1)$ using S.L.Sobolev's method for any number $N+1$ of the nodes $x_{\beta}$, i.e. finding the coefficients $C_{\beta}$ satisfying the following equality

$$
\left\|\ell(x)\left|W_{2}^{(m, m-1) *}(0,1)\left\|=\inf _{C_{\beta}}\right\| \ell(x)\right| W_{2}^{(m, m-1) *}(0,1)\right\| .
$$

Thus, in order to construct the optimal quadrature formula in the sense of Sard in the space $W_{2}^{(m, m-1)}(0,1)$ we need consequently to solve the following problems.

Problem 1. Find the norm of the error functional $\ell(x)$ of quadrature formulas (1.1) in the space $W_{2}^{(m, m-1) *}(0,1)$.

Problem 2. Find the coefficients $C_{\beta}$ which satisfy equality (1.5) when the nodes $x_{\beta}$ are fixed.

The structure of the present paper is: in the second section the extremal function which corresponds to the error functional $\ell(x)$ is found and with its help representation of the norm of the error functional (1.2) is calculated, i.e. the problem 1 is solved; in the third section in order to find the minimum of the quantity $\|\ell\|^{2}$ by coefficients $C_{\beta}$ the system of linear equations is obtained for the coefficients of optimal quadrature formula in the sense of Sard in the space $W_{2}^{(m, m-1)}(0,1)$, moreover existence 
and uniqueness of the solution of this system are proved; in the forth section explicit formulas for the coefficients of optimal quadrature formula of the form (1.1) are found, i.e. the problem 2 is solved; finally, in the fifth section the results of numerical experiments are given.

\section{The EXtRemal FUnCTION AND THE REPRESENTATION OF THE NORM OF THE ERROR FUNCTIONAL $\ell(x)$}

In order to solve the problem 1, i.e. for calculation the norm of the error functional (1.2) in the space $W_{2}^{(m, m-1) *}(0,1)$, it is used the concept of extremal function of given functional. The function $\psi_{\ell}(x)$ is called the extremal function for the functional $\ell(x)$ (see, [28]), if the following equality is fulfilled

$$
\left(\ell(x), \psi_{\ell}(x)\right)=\left\|\ell(x)\left|W_{2}^{(m, m-1) *}(0,1)\|\cdot\| \psi_{\ell}(x)\right| W_{2}^{(m, m-1)}(0,1)\right\| \cdot
$$

This means that the extremal function $\psi_{\ell}(x)$ gives the greatest error to the difference (1.4) in the space $W_{2}^{(m, m-1)}(0,1)$.

Since the space $W_{2}^{(m, m-1)}(0,1)$ is Hilbert space then the extremal function $\psi_{\ell}(x)$ in this space is found with the help of Riesz theorem about general form of a linear continuous functional on Hilbert spaces. Then for functional $\ell(x)$ and for any $\varphi(x) \in W_{2}^{(m, m-1)}(0,1)$ there exists the function $\psi_{\ell}(x) \in W_{2}^{(m, m-1)}(0,1)$ for which the following equation is take placed

$$
(\ell(x), \varphi(x))=\left\langle\psi_{\ell}(x), \varphi(x)\right\rangle,
$$

where

$$
\left\langle\psi_{\ell}(x), \varphi(x)\right\rangle=\int_{0}^{1}\left(\psi_{\ell}^{(m)}(x)+\psi_{\ell}^{(m-1)}(x)\right)\left(\varphi^{(m)}(x)+\varphi^{(m-1)}(x)\right) d x
$$

is the inner product defined in the space $W_{2}^{(m, m-1)}(0,1)$.

Further, we will solve equation (2.2).

Suppose $\varphi(x)$ belongs to the space $\stackrel{\circ}{C}^{(\infty)}(0,1)$, where $\stackrel{\circ}{C}^{(\infty)}(0,1)$ is the space of functions, which are infinity differentiable and finite in the interval $(0,1)$. Then from $(2.3)$, integrating by parts, we obtain

$$
\left\langle\psi_{\ell}(x), \varphi(x)\right\rangle=(-1)^{m} \int_{0}^{1}\left(\psi_{\ell}^{(2 m)}(x)-\psi_{\ell}^{(2 m-2)}(x)\right) \varphi(x) d x .
$$

Keeping in mind (2.4) from (2.2) we get

$$
\psi_{\ell}^{(2 m)}(x)-\psi_{\ell}^{(2 m-2)}(x)=(-1)^{m} \ell(x) .
$$

So, when $\varphi(x) \in \stackrel{\circ}{C}^{(\infty)}(0,1)$ the extremal function $\psi_{\ell}(x)$ is a solution of equation $(2.5)$. But, we have to find the solution of equation $(2.2)$ when $\varphi(x) \in W_{2}^{(m, m-1)}(0,1)$. Since the space $\stackrel{\circ}{C}(0,1)$ is densely in the space $W_{2}^{(m, m-1)}(0,1)$, then we can approximate arbitrarily exact functions of the space $W_{2}^{(m, m-1)}(0,1)$ by a sequence of functions of the space $\stackrel{\circ}{C}(0,1)$. Next for any $\varphi(x) \in W_{2}^{(m, m-1)}(0,1)$ we consider the inner product $\left\langle\psi_{\ell}(x), \varphi(x)\right\rangle$ and, integrating by parts, we have

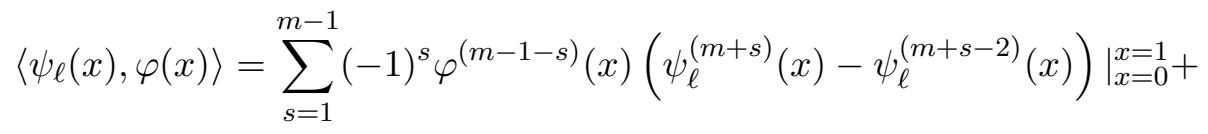




$$
+\left.\varphi^{(m-1)}(x)\left(\psi_{\ell}^{(m)}(x)+\psi_{\ell}^{(m-1)}(x)\right)\right|_{x=0} ^{x=1}+(-1)^{m} \int_{0}^{1} \varphi(x)\left(\psi_{\ell}^{(2 m)}(x)-\psi_{\ell}^{(2 m-2)}(x)\right) d x .
$$

Hence from arbitrariness $\varphi(x)$ and uniqueness of the function $\psi_{\ell}(x)$ (up to the function $e^{-x}$ and polynomial of degree $m-2$ ), taking into account (2.5), it must be fulfilled the following equations

$$
\begin{gathered}
\psi_{\ell}^{(2 m)}(x)-\psi_{\ell}^{(2 m-2)}(x)=(-1)^{m} \ell(x), \\
\left.\left(\psi_{\ell}^{(m+s)}(x)-\psi_{\ell}^{(m+s-2)}(x)\right)\right|_{x=0} ^{x=1}=0, \\
\left.\left(\psi_{\ell}^{(m)}(x)+\psi_{\ell}^{(m-1)}(x)\right)\right|_{x=0} ^{x=1}=0 .
\end{gathered}
$$

Thus, we conclude that the extremal function $\psi_{\ell}(x)$ is the solution of the boundary value problem (2.6)-(2.8).

The following holds

Theorem 2.1. The solution of the boundary value problem (2.6)-(2.8) is the extremal function $\psi_{\ell}(x)$ of the error functional $\ell(x)$ and has the following form

$$
\psi_{\ell}(x)=(-1)^{m} \ell(x) * G(x)+P_{m-2}(x)+d e^{-x},
$$

where

$$
G(x)=\frac{\operatorname{sign} x}{2}\left(\frac{e^{x}-e^{-x}}{2}-\sum_{k=1}^{m-1} \frac{x^{2 k-1}}{(2 k-1) !}\right)
$$

is a solution of the equation

$$
\psi^{(2 m)}(x)-\psi^{(2 m-2)}(x)=\delta(x)
$$

$d$ is any real number, $P_{m-2}(x)$ is a polynomial of degree $m-2$.

Proof. It is known that general solution of nonhomogeneous differential equation consists on sum of a partial solution of nonhomogeneous differential equation and general solution of corresponding homogeneous differential equation.

The homogeneous equation for differential equation (2.6) has the form

$$
\psi_{\ell}^{(2 m)}(x)-\psi_{\ell}^{(2 m-2)}(x)=0 .
$$

It is easy to show that general solution of equation (2.11) is

$$
P_{2 m-3}(x)+d_{1} e^{x}+d_{2} e^{-x} .
$$

It is not difficult to verify that a partial solution of the differential equation (2.6) is

$$
(-1)^{m} \ell(x) * G(x),
$$

where $G(x)$ is a fundamental solution of equation (2.6) and is defined by (2.9) and is a solution of equation (2.10), * is operation of convolution, where convolution of two functions is defined as

$$
f(x) * g(x)=\int_{-\infty}^{\infty} f(x-y) g(y) d y=\int_{-\infty}^{\infty} f(y) g(x-y) d y .
$$

The rule of finding a fundamental solution of a linear differential operator

$$
L \equiv \frac{d^{n}}{d x^{n}}+a_{1} \frac{d^{n-1}}{d x^{n-1}}+\ldots+a_{n}
$$


where $a_{j}$ are constants, is given in [30, p.88]. Using this rule, it is found the function $G(x)$, which is a fundamental solution of the operator $\frac{d^{2 m}}{d x^{2 m}}-\frac{d^{2 m-2}}{d x^{2 m-2}}$ and have the form $(2.9)$.

Thus, we have the following general solution of the equation $(2.6)$

$$
\psi_{\ell}(x)=(-1)^{m} \ell(x) * G(x)+P_{2 m-3}(x)+d_{1} e^{x}+d_{2}^{-x},
$$

where $P_{2 m-3}(x)=b_{2 m-3} x^{2 m-3}+\ldots+b_{1} x+b_{0}$ is a polynomial of degree $2 m-3, d_{1}, d_{2}$ are constants.

In order that in the space $W_{2}^{(m, m-1)}(0,1)$ the function $\psi_{\ell}(x)$ will be unique (up to the function $e^{-x}$ and polynomial of degree $m-2)$, it has to satisfies the conditions (2.7), (2.8). Here derivative is in generalized sense and

$$
\begin{gathered}
\psi_{\ell}^{(k)}(x)=(-1)^{m} \ell(x) * G^{(k)}(x)+P_{2 m-3}^{(k)}(x)+d_{1} e^{x}+(-1)^{k} d_{2} e^{-x}, \\
k=1,2, \ldots, 2 m-1,
\end{gathered}
$$

where

$$
G^{(k)}(x)=\frac{\operatorname{sign} x}{2}\left\{\begin{array}{l}
\frac{e^{x}-e^{-x}}{2}-\frac{x^{2 m-3-k}}{(2 m-3-k) !}-\ldots-\frac{x^{3}}{3 !}-x \text { when } k \text { is even, } \\
\frac{e^{x}+e^{-x}}{2}-\frac{x^{2 m-3-k}}{(2 m-3-k) !}-\ldots-\frac{x^{2}}{2 !}-1 \text { when } k \text { is odd. }
\end{array}\right.
$$

From conditions (2.7) for $s=m-1$, taking into account

we get

$$
\begin{gathered}
\psi_{\ell}^{(2 m-1)}(x)=(-1)^{m} \ell(x) *\left\{\operatorname{sign} x \cdot \frac{e^{x}+e^{-x}}{4}\right\}+d_{1} e^{x}-d_{1} e^{-x}, \\
\psi_{\ell}^{(2 m-3)}(x)=(-1)^{m} \ell(x) *\left\{\frac{\operatorname{sign} x}{2}\left[\frac{e^{x}+e^{-x}}{2}-1\right]\right\}+(2 m-3) ! b_{2 m-3}+d_{1} e^{x}-d_{1} e^{-x},
\end{gathered}
$$

$$
\begin{gathered}
\psi_{\ell}^{(2 m-1)}(x)-\psi_{\ell}^{(2 m-3)}(x)=(-1)^{m} \ell(x) * \frac{\operatorname{sign} x}{2}-(2 m-3) ! b_{2 m-3}= \\
=(-1)^{m}\left(\ell(y), \frac{\operatorname{sign}(x-y)}{2}\right)-(2 m-3) ! b_{2 m-3} .
\end{gathered}
$$

Hence for $x=0$ :

$$
\begin{gathered}
\psi_{\ell}^{(2 m-1)}(0)-\psi_{\ell}^{(2 m-3)}(0)=(-1)^{m}\left(\ell(y), \frac{\operatorname{sign}(-y)}{2}\right)-(2 m-3) ! b_{2 m-3}= \\
=-\frac{(-1)^{m}}{2}(\ell(y), 1)-(2 m-3) ! b_{2 m-3}=0
\end{gathered}
$$

for $x=1$ :

Then

$$
\begin{gathered}
\psi_{\ell}^{(2 m-1)}(1)-\psi_{\ell}^{(2 m-3)}(1)=(-1)^{m}\left(\ell(y), \frac{\operatorname{sign}(1-y)}{2}\right)-(2 m-3) ! b_{2 m-3}= \\
=\frac{(-1)^{m}}{2}(\ell(y), 1)-(2 m-3) ! b_{2 m-3}=0 .
\end{gathered}
$$

$$
b_{2 m-3}=0 \quad \text { and } \quad(\ell(y), 1)=0 .
$$

When $s=m-2$ from conditions (2.7), taking into account

$$
\psi_{\ell}^{(2 m-2)}(x)=(-1)^{m} \ell(x) *\left\{\operatorname{sign} x \cdot \frac{e^{x}-e^{-x}}{4}\right\}+d_{1} e^{x}+d_{1} e^{-x},
$$


we have

$$
\begin{gathered}
\psi_{\ell}^{(2 m-4)}(x)=(-1)^{m} \ell_{N}(x) *\left\{\frac{\operatorname{sign} x}{2}\left[\frac{e^{x}-e^{-x}}{2}-x\right]\right\}+ \\
+(2 m-3) ! b_{2 m-3} x+(2 m-4) ! b_{2 m-4}+d_{1} e^{x}+d_{2} e^{-x}
\end{gathered}
$$

$$
\psi_{\ell}^{(2 m-2)}(x)-\psi_{\ell}^{(2 m-4)}(x)=(-1)^{m} \ell(x) * \frac{x \cdot \operatorname{sign} x}{2}-(2 m-3) ! b_{2 m-3} x-(2 m-4) ! b_{2 m-4} .
$$

Hence, taking account of (2.14), for $x=0$ :

for $x=1$ :

$$
\begin{gathered}
(-1)^{m}\left(\ell(y), \frac{0-y}{2} \operatorname{sign}(0-y)\right)-(2 m-4) ! b_{2 m-4}= \\
=\frac{(-1)^{m}}{2}(\ell(y), y)-(2 m-4) ! b_{2 m-4}=0,
\end{gathered}
$$

Hence

$$
\begin{gathered}
(-1)^{m}\left(\ell(y), \frac{1-y}{2} \operatorname{sign}(1-y)\right)-(2 m-4) ! b_{2 m-4}= \\
=-\frac{(-1)^{m}}{2}(\ell(y), y)-(2 m-4) ! b_{2 m-4}=0 .
\end{gathered}
$$

$$
b_{2 m-4}=0 \text { and }(\ell(y), y)=0
$$

and so on, continuing by this manner, for $s=m-3, m-4, \ldots, 2,1$ we obtain

$$
b_{m-2+s}=0, \quad\left(\ell(y), y^{m-1-s}\right)=0 .
$$

Combining (2.14), (2.15), (2.16) we get

$$
\begin{gathered}
b_{2 m-3-s}=0, \quad s=0,1, \ldots, m-2, \\
\left(\ell(y), y^{s}\right), \quad s=0,1, \ldots, m-2 .
\end{gathered}
$$

From the condition (2.8), keeping in mind (2.17), we have

Hence

$$
\begin{gathered}
\psi_{\ell}^{(m)}(x)=(-1)^{m} \ell(x) * G^{(m)}(x)+d_{1} e^{x}+(-1)^{m} d_{2} e^{-x}, \\
\psi_{\ell}^{(m-1)}(x)=(-1)^{m} \ell(x) * G^{(m-1)}(x)+d_{1} e^{x}+(-1)^{m-1} d_{2} e^{-x} .
\end{gathered}
$$

$$
\begin{gathered}
\psi_{\ell}^{(m)}(x)+\psi_{\ell}^{(m-1)}(x)= \\
=(-1)^{m} \ell(x) *\left\{\frac{\operatorname{sign} x}{2}\left(e^{x}-\frac{x^{m-2}}{(m-2) !}-\frac{x^{m-3}}{(m-3) !}-\ldots-x-1\right)\right\}+d_{1} e^{x} .
\end{gathered}
$$

For $x=0$, taking into account (2.18),

$$
\begin{gathered}
\psi_{\ell}^{(m)}(0)+\psi_{\ell}^{(m-1)}(0)=(-1)^{m}\left(\ell(y), \frac{\operatorname{sign}(0-y)}{2} \cdot\left(e^{-y}-\frac{(-y)^{m-2}}{(m-2) !}-\right.\right. \\
\left.\left.-\frac{(-y)^{m-3}}{(m-3) !}-\ldots+y-1\right)\right)+d_{1}=-\frac{(-1)^{m}}{2}\left(\ell_{N}(y), e^{-y}\right)+d_{1}=0
\end{gathered}
$$

For $x=1$ :

$$
\psi_{\ell}^{(m)}(1)+\psi_{\ell}^{(m-1)}(1)=(-1)^{m}\left(\ell(y), \frac{\operatorname{sign}(1-y)}{2} \cdot\left(e^{1-y}-\frac{(1-y)^{m-2}}{(m-2) !}-\right.\right.
$$


Whence

$$
\left.\left.-\frac{(1-y)^{m-3}}{(m-3) !}-\ldots-(1-y)-1\right)\right)+d_{1} e=\frac{(-1)^{m} e}{2}\left(\ell_{N}(y), e^{-y}\right)+d_{1} e=0
$$

$$
\begin{gathered}
d_{1}=0, \\
\left(\ell(y), e^{-y}\right)=0 .
\end{gathered}
$$

Taking into account equalities (2.17)- (2.20) and denoting $d_{2}=d$, we get the statement of the theorem. Theorem 2.1 is proved.

The equalities (2.18) and (2.20) mean that our quadrature formula is exact for the function $e^{-x}$ and for any polynomial of degree up to $m-2$.

Now, using the result of theorem 2.1, we immediately obtain the representation of square of the norm of the error functional (1.2)

$$
\begin{gathered}
\left\|\ell(x) \mid W_{2}^{(m, m-1) *}(0,1)\right\|^{2}=\left(\ell(x), \psi_{\ell}(x)\right)=(-1)^{m}\left[\sum_{\beta=0}^{N} \sum_{\gamma=0}^{N} C_{\beta} C_{\gamma} G\left(x_{\beta}-x_{\gamma}\right)-\right. \\
\left.-2 \sum_{\beta=0}^{N} C_{\beta} \int_{0}^{1} G\left(x-x_{\beta}\right) d x+\int_{0}^{1} \int_{0}^{1} G(x-y) d x d y\right],
\end{gathered}
$$

Thus the problem 1 is solved.

Further in sections 3 and 4 we solve the problem 2.

\section{The SYSTEM FOR THE COEFFICIENTS OF OPTIMAL QUADRATURE FORMUlas IN THE SPACE}

$$
W_{2}^{(m, m-1)}(0,1)
$$

Assume that the nodes $x_{\beta}$ of the quadrature formula (1.1) are fixed. The error functional (1.2) satisfies conditions (2.18) and (2.20). The norm of the error functional $\ell(x)$ is multidimensional function with respect to the coefficients $C_{\beta}(\beta=\overline{0, N})$. For finding the point of conditional minimum of the expression (2.21) under the conditions (2.18) and (2.20) we apply the method of undetermined multipliers of Lagrange.

We denote $\mathbf{C}=\left(C_{0}, C_{1}, \ldots, C_{N}\right)$ and $\lambda=\left(\lambda_{0}, \lambda_{1}, \ldots, \lambda_{m-1}\right)$.

Consider the function

$$
\Psi(\mathbf{C}, \lambda)=\|\ell(x)\|^{2}-2(-1)^{m} \sum_{\alpha=0}^{m-2} \lambda_{\alpha}\left(\ell(x), x^{\alpha}\right)-2(-1)^{m} \lambda_{m-1}\left(\ell(x), e^{-x}\right) .
$$

Equating to 0 partial derivatives of $\Psi(\mathbf{C}, \lambda)$ by $C_{\beta}(\beta=\overline{0, N})$ and $\lambda_{0}, \lambda_{1}, \ldots, \lambda_{m-1}$, we get the following linear system

$$
\begin{gathered}
\sum_{\gamma=0}^{N} C_{\gamma} G\left(x_{\beta}-x_{\gamma}\right)+\sum_{\alpha=0}^{m-2} \lambda_{\alpha} x_{\beta}^{\alpha}+\lambda_{m-1} e^{-x_{\beta}}=f_{m}\left(x_{\beta}\right), \quad \beta=\overline{0, N} \\
\sum_{\gamma=0}^{N} C_{\gamma} x_{\gamma}^{\alpha}=\frac{1}{\alpha+1}, \alpha=0,1, \ldots, m-2, \\
\sum_{\gamma=0}^{N} C_{\gamma} e^{-x_{\gamma}}=1-e^{-1},
\end{gathered}
$$


where $G(x)$ is defined by equality $(2.9)$,

$$
f_{m}\left(x_{\beta}\right)=\int_{0}^{1} G\left(x-x_{\beta}\right) d x .
$$

The system (3.1)-(3.3) has a unique solution and this solution gives minimum to $\|\ell(x)\|^{2}$ under the conditions (3.2), (3.3). Uniqueness of the solution of such type of systems is discussed in [28, 29].

It should be noted that existence and uniqueness of optimal quadrature formulas in the sense of Sard is also investigated in [12].

Now in (2.21) we will do change of variables $C_{\beta}=\bar{C}_{\beta}+C_{1 \beta}$. Then (2.21) and the system (3.1)-(3.3) have the following form:

$$
\begin{gathered}
\|\ell\|^{2}=(-1)^{m}\left[\sum_{\beta=0}^{N} \sum_{\gamma=0}^{N} \bar{C}_{\beta} \bar{C}_{\gamma} G\left(x_{\beta}-x_{\gamma}\right)-2 \sum_{\beta=0}^{N}\left(\bar{C}_{\beta}+C_{1, \beta}\right) \int_{0}^{1} G\left(x-x_{\beta}\right) d x+\right. \\
\left.+\sum_{\beta=0}^{N} \sum_{\gamma=0}^{N}\left(2 \bar{C}_{\beta} C_{1, \gamma}+C_{1, \beta} C_{1, \gamma}\right) G\left(x_{\beta}-x_{\gamma}\right)+\int_{0}^{1} \int_{0}^{1} G(x-y) d x d y\right], \\
\sum_{\gamma=0}^{N} \bar{C}_{\gamma} G\left(x_{\beta}-x_{\gamma}\right)+\sum_{\alpha=0}^{m-2} \lambda_{\alpha} x_{\beta}^{\alpha}+\lambda_{m-1} e^{-x_{\beta}}=F_{m}\left(x_{\beta}\right), \quad \beta=\overline{0, N} \\
\sum_{\gamma=0}^{N} \bar{C}_{\gamma} x_{\gamma}^{\alpha}=0, \quad \alpha=\overline{0, m-2}, \\
\sum_{\gamma=0}^{N} \bar{C}_{\gamma} e^{-x_{\gamma}}=0,
\end{gathered}
$$

where $F_{m}\left(x_{\beta}\right)=f_{m}\left(x_{\beta}\right)-\sum_{\gamma=0}^{N} C_{1, \gamma} G\left(x_{\beta}-x_{\gamma}\right), C_{1 \beta}$ is a partial solution of equations (3.2), (3.3).

Hence we directly get that the minimization of (2.21) under the conditions (3.2), (3.3) with respect to $C_{\beta}$ is equivalent to the minimization of expression (3.5) with respect to $\bar{C}_{\beta}$ under the conditions (3.7), (3.8). Therefore it is sufficient to prove that the system (3.6)-(3.8) has a unique solution with respect to unknowns $\overline{\mathbf{C}}=\left(\bar{C}_{0}, \bar{C}_{1}, \ldots, \bar{C}_{N}\right), \lambda=\left(\lambda_{0}, \lambda_{1}, \ldots, \lambda_{m-1}\right)$ and this solution gives conditional minimum to the expression $\|\ell\|^{2}$.

From the theory of conditional extremum it is known the sufficient condition in which the solution of the system (3.6) - (3.8) gives conditional minimum to the expression $\|\ell\|^{2}$ on the manifold (3.7), (3.8). It consists on positiveness of the quadratic form

$$
\Phi(\overline{\mathbf{C}})=\sum_{\beta=0}^{N} \sum_{\gamma=0}^{N} \frac{\partial^{2} \Psi}{\partial \bar{C}_{\beta} \partial \bar{C}_{\gamma}} \bar{C}_{\beta} \bar{C}_{\gamma}
$$

on the set of the vectors $\overline{\mathbf{C}}=\left(\bar{C}_{0}, \bar{C}_{1}, \ldots, \bar{C}_{N}\right)$ under the condition

$$
S \overline{\mathbf{C}}=0
$$


where $S$ is the following matrix of equations (3.7), (3.8):

$$
S=\left(\begin{array}{cccc}
1 & 1 & \cdots & 1 \\
x_{0} & x_{1} & \cdots & x_{N} \\
\vdots & \vdots & \ddots & \vdots \\
x_{0}^{m-2} & x_{1}^{m-2} & \cdots & x_{N}^{m-2} \\
e^{-x_{0}} & e^{-x_{1}} & \cdots & e^{-x_{N}}
\end{array}\right)
$$

We will show that in our case this condition is fulfilled.

Theorem 3.1. For any nonzero vector $\overline{\mathbf{C}} \in R^{N+1}$ lying in the subspace $S \overline{\mathbf{C}}=0$, the function $\Phi(\overline{\mathbf{C}})$ is strictly positive.

Proof. Using the definition of the function $\Psi(\mathbf{C}, \lambda)$ and equations (3.5), (3.7), (3.8) from (3.9) we get

$$
\Phi(\overline{\mathbf{C}})=2(-1)^{m} \sum_{\beta=0}^{N} \sum_{\gamma=0}^{N} G\left(x_{\beta}-x_{\gamma}\right) \bar{C}_{\beta} \bar{C}_{\gamma} .
$$

Consider the linear combination of delta functions

$$
\delta_{\overline{\mathbf{C}}}(x)=\sqrt{2} \sum_{\beta=0}^{N} \bar{C}_{\beta} \delta\left(x-x_{\beta}\right)
$$

By virtue of the condition (3.10) this functional belongs to the space $W_{2}^{(m, m-1) *}(0,1)$. So, it has the extremal function $u_{\overline{\mathbf{C}}}(x) \in W_{2}^{(m, m-1)}(0,1)$ which is a solution of the equation

$$
\left(\frac{d^{2 m}}{d x^{2 m}}-\frac{d^{2 m-2}}{d x^{2 m-2}}\right) u_{\overline{\mathbf{C}}}(x)=(-1)^{m} \delta_{\overline{\mathbf{C}}}(x) .
$$

As $u_{\overline{\mathbf{C}}}(x)$ we can take linear combination of shifts of the fundamental solution $G(x)$ :

$$
u_{\overline{\mathbf{C}}}(x)=\sqrt{2}(-1)^{m} \sum_{\beta=0}^{N} \bar{C}_{\beta} G\left(x-x_{\beta}\right) .
$$

The square of its norm in the space $W_{2}^{(m, m-1)}(0,1)$ coincide with $\Phi(\overline{\mathbf{C}})$, i.e.

$$
\left\|u_{\overline{\mathbf{C}}}(x) \mid W_{2}^{(m, m-1)}(0,1)\right\|^{2}=\left(\delta_{\overline{\mathbf{C}}}(x), u_{\overline{\mathbf{C}}}(x)\right)=2(-1)^{m} \sum_{\beta=0}^{N} \sum_{\gamma=0}^{N} \bar{C}_{\beta} \bar{C}_{\gamma} G\left(x_{\beta}-x_{\gamma}\right) .
$$

Hence clearly that for nonzero $\overline{\mathbf{C}}$ the function $\Phi(\overline{\mathbf{C}})$ is strictly positive.

Theorem 3.1 is proved.

If the nodes $x_{0}, x_{1}, \ldots, x_{N}$ are selected such that the matrix $S$ has right inverse matrix, then the system (3.6) - (3.8) has a unique solution. Then the system (3.1)-(3.3) also has a unique solution.

Theorem 3.2. If the matrix $S$ has right inverse matrix, then the main matrix $Q$ of the system (3.6) - (3.8) is nonsingular.

Proof. We denote by $M$ the matrix of quadratic form $\frac{(-1)^{m}}{2} \Phi(\overline{\mathbf{C}})$, where $\Phi(\overline{\mathbf{C}})$ is defined by equality (3.11). It is known that if homogenous system of linear equations has only trivial solution 
then corresponding nonhomogeneous system has a unique solution. Consider homogeneous system corresponding to the system (3.6) -(3.8) in the following matrix form:

$$
Q\left(\begin{array}{c}
\overline{\mathbf{C}} \\
\lambda
\end{array}\right)=\left(\begin{array}{cc}
M & S^{*} \\
S & 0
\end{array}\right)\left(\begin{array}{c}
\overline{\mathbf{C}} \\
\lambda
\end{array}\right)=0
$$

where $S^{*}$ is the transposed matrix to the matrix $S$.

We verify, that unique solution of (3.14) is identical zero. Suppose $\overline{\mathbf{C}}, \lambda$ is the solution of (3.14). Consider the function $\delta_{\overline{\mathbf{C}}}(x)$, which is determined by equality (3.12). As the extremal function for the function $\delta_{\overline{\mathbf{C}}}(x)$ we take the following function:

$$
u_{\overline{\mathbf{C}}}(x)=\sqrt{2}(-1)^{m}\left(\sum_{\beta=0}^{N} \bar{C}_{\beta} G\left(x-x_{\beta}\right)+\sum_{\alpha=0}^{m-2} \lambda_{\alpha} x^{\alpha}+\lambda_{m-1} e^{-x}\right) .
$$

This is possible because the function $u_{\overline{\mathbf{C}}}(x)$ belongs to the space $W_{2}^{(m, m-1)}(0,1)$ and is a solution of equation (3.13). First $N+1$ equations of the system (3.14) mean that $u_{\overline{\mathbf{C}}}(x)$ takes 0 values at all nodes $x_{\beta}$. Then for the norm of the functional $\delta_{\overline{\mathbf{C}}}(x)$ in $W_{2}^{(m, m-1) *}(0,1)$ we have

$$
\left\|\delta_{\overline{\mathbf{C}}}(x) \mid W_{2}^{(m, m-1) *}(0,1)\right\|^{2}=\left(\delta_{\overline{\mathbf{C}}}(x), u_{\overline{\mathbf{C}}}(x)\right)=2(-1)^{m} \sum_{\beta=0}^{N} \bar{C}_{\beta} u_{\overline{\mathbf{C}}}\left(x_{\beta}\right)=0
$$

On the other hand taking into account equations (3.7), (3.8) we get

$$
\left\|\delta_{\overline{\mathbf{C}}}(x) \mid W_{2}^{(m, m-1) *}(0,1)\right\|^{2}=\left(\delta_{\overline{\mathbf{C}}}(x), u \overline{\mathbf{C}}(x)\right)=2(-1)^{m} \sum_{\beta=0}^{N} \sum_{\gamma=0}^{N} \bar{C}_{\beta} \bar{C}_{\gamma} G\left(x_{\beta}-x_{\gamma}\right) .
$$

From (3.16) we conclude that (3.15) is possible if $\overline{\mathbf{C}}=0$. Then from first $N+1$ equations of the system (3.14) we obtain

$$
S^{*} \lambda=0 .
$$

By assertion of the theorem, the matrix $S$ has right inverse matrix, then $S^{*}$ has left inverse matrix. Then from (3.17) we conclude that the solution $\lambda$ also is equal to zero.

Theorem 3.2 is proved.

From (2.21) and theorems 3.1, 3.2 it follows that in fixed values of the nodes $x_{\beta}$ the square of the norm of the error functional $\ell(x)$ being quadratic functions of the coefficients $C_{\beta}$ has a unique minimum in some concrete value $C_{\beta}=\stackrel{\circ}{C}_{\beta}$.

As said in the first section the quadrature formulas with the coefficients $\stackrel{\circ}{C}_{\beta} \quad(\beta=\overline{0, N})$, corresponding to this minimum in fixed nodes $x_{\beta}$ is called optimal quadrature formula in the sense of Sard and $\stackrel{\circ}{C}_{\beta}(\beta=\overline{0, N})$ are called optimal coefficients.

Below for convenience the optimal coefficients $\stackrel{\circ}{C}_{\beta}$ remain as $C_{\beta}$.

\section{Coefficients of optimal quadrature formula in the Sense of Sard}

In the present section we will solve the system (3.1)-(3.3) and will find explicit formula for the coefficients $C_{\beta}$. Here we will use similar method suggested by S.L.Sobolev [27] for finding the coefficients of optimal quadrature formulas in the space $L_{2}^{(m)}(0,1)$. Here mainly is used the concept of functions of 
discrete argument and operations on them. Theory of discrete argument functions is given in [28,29]. For completeness we give some definitions about functions of discrete argument.

Assume that the nodes $x_{\beta}$ are equal spaced, i.e. $x_{\beta}=h \beta, h=\frac{1}{N}, N=1,2, \ldots$.

Suppose that $\varphi(x)$ and $\psi(x)$ are real-valued functions of real variable and are defined in real line $\mathbb{R}$.

Definition 4.1. A function $\varphi(h \beta)$ is called function of discrete argument if it is given on some set of integer values of $\beta$.

Definition 4.2. By inner product of two discrete functions $\varphi(h \beta)$ and $\psi(h \beta)$ is called the number

$$
[\varphi(h \beta), \psi(h \beta)]=\sum_{\beta=-\infty}^{\infty} \varphi(h \beta) \cdot \psi(h \beta),
$$

if the series on the right hand side of the last equality converges absolutely.

Definition 4.3. By convolution of two discrete functions $\varphi(h \beta)$ and $\psi(h \beta)$ is called the inner product

$$
\varphi(h \beta) * \psi(h \beta)=[\varphi(h \gamma), \psi(h \beta-h \gamma)]=\sum_{\gamma=-\infty}^{\infty} \varphi(h \gamma) \cdot \psi(h \beta-h \gamma) .
$$

Now we turn on to our problem.

Suppose that $C_{\beta}=0$ when $\beta<0$ and $\beta>N$. Using above mentioned definitions the system (3.1)-(3.3) we rewrite in the convolution form

$$
\begin{gathered}
G(h \beta) * C_{\beta}+P_{m-2}(h \beta)+d e^{-h \beta}=f_{m}(h \beta), \quad \beta=0,1, \ldots, N \\
C_{\beta}=0, \text { when } \quad \beta<0 \text { and } \beta>N, \\
\sum_{\beta=0}^{N} C_{\beta} \cdot(h \beta)^{\alpha}=\frac{1}{\alpha+1}, \quad \alpha=0,1, \ldots, m-2, \\
\sum_{\beta=0}^{N} C_{\beta} \cdot e^{-h \beta}=1-e^{-1},
\end{gathered}
$$

where

$$
f_{m}(h \beta)=\frac{e^{h \beta}+e^{-h \beta}+e^{1-h \beta}+e^{h \beta-1}-4}{4}-\sum_{k=1}^{m-1} \frac{(h \beta)^{2 k}+(1-h \beta)^{2 k}}{2 \cdot(2 k) !} .
$$

Consider the following problem.

Problem A. Find the discrete function $C_{\beta}$, polynomial $P_{m-2}(h \beta)$ of degree $m-2$ and unknown constant $d$ which satisfy the system (4.1)-(4.4) for given $f_{m}(h \beta)$.

Further we investigate the problem $\mathrm{A}$ and instead of $C_{\beta}$ we introduce the functions

$$
v(h \beta)=G(h \beta) * C_{\beta}
$$

and

$$
u(h \beta)=v(h \beta)+P_{m-2}(h \beta)+d e^{-h \beta} .
$$

In such statement it is necessary to express the coefficients $C_{\beta}$ by the function $u(h \beta)$. For this we have to construct such operator $D_{m}(h \beta)$ which satisfies the equality

$$
D_{m}(h \beta) * G(h \beta)=\delta(h \beta),
$$

where $\delta(h \beta)$ is equal to 0 when $\beta \neq 0$ and is equal to 1 when $\beta=0$, i.e. $\delta(h \beta)$ is the discrete delta-function. 
In connection with this in $[21,22]$ the discrete analogue $D_{m}(h \beta)$ of the operator $\frac{d^{2 m}}{d x^{2 m}}-\frac{d^{2 m-2}}{d x^{2 m-2}}$, which satisfies equation (4.8) is constructed and its some properties are investigated.

The following theorems are proved in the works $[21,22]$.

Theorem 4.1. The discrete analogue of the differential operator $\frac{d^{2 m}}{d x^{2 m}}-\frac{d^{2 m-2}}{d x^{2 m-2}}$ satisfying the equation (4.8) has the form

$$
D_{m}(h \beta)=\frac{1}{p_{2 m-2}^{(2 m-2)}} \begin{cases}\sum_{k=1}^{m-1} A_{k} \lambda_{k}^{|\beta|-1}, & |\beta| \geq 2, \\ -2 e^{h}+\sum_{k=1}^{m-1} A_{k}, & |\beta|=1, \\ 2 C+\sum_{k=1}^{m-1} \frac{A_{k}}{\lambda_{k}}, & \beta=0\end{cases}
$$

where

$$
\begin{gathered}
C=1+(2 m-2) e^{h}+e^{2 h}+\frac{e^{h} \cdot p_{2 m-3}^{(2 m-2)}}{p_{2 m-2}^{(2 m-2)}}, \\
A_{k}=\frac{2\left(1-\lambda_{k}\right)^{2 m-2}\left[\lambda_{k}\left(e^{2 h}+1\right)-e^{h}\left(\lambda_{k}^{2}+1\right)\right] p_{2 m-2}^{(2 m-2)}}{\lambda_{k} P_{2 m-2}^{\prime}\left(\lambda_{k}\right)}, \\
\mathcal{P}_{2 m-2}(\lambda)=\sum_{s=0}^{2 m-2} p_{s}^{(2 m-2)} \lambda^{s}=\left(1-e^{2 h}\right)(1-\lambda)^{2 m-2}-2\left(\lambda\left(e^{2 h}+1\right)-e^{h}\left(\lambda^{2}+1\right)\right) \times \\
\times\left[h(1-\lambda)^{2 m-4}+\frac{h^{3}(1-\lambda)^{2 m-6}}{3 !} E_{2}(\lambda)+\ldots+\frac{h^{2 m-3} E_{2 m-4}(\lambda)}{(2 m-3) !}\right],
\end{gathered}
$$

$p_{2 m-2}^{(2 m-2)}, p_{2 m-3}^{(2 m-2)}$ are the coefficients of the polynomial $\mathcal{P}_{2 m-2}(\lambda)$ defined by equality (4.12), $\lambda_{k}$ are the roots of the polynomial $\mathcal{P}_{2 m-2}(\lambda)$ which absolute values less than $1, E_{k}(\lambda)$ is the Euler-Frobenius polynomial of degree $k$ (see [29]).

Theorem 4.2. The discrete analogue $D_{m}(h \beta)$ of the differential operator $\frac{d^{2 m}}{d x^{2 m}}-\frac{d^{2 m-2}}{d x^{2 m-2}}$ satisfies the following equalities

1) $D_{m}(h \beta) * e^{h \beta}=0$,

2) $D_{m}(h \beta) * e^{-h \beta}=0$,

3) $D_{m}(h \beta) *(h \beta)^{n}=0, n \leq 2 m-3$,

4) $D_{m}(h \beta) * G(h \beta)=\delta(h \beta)$,

here $G(h \beta)$ is the function of discrete argument corresponding to the function $G(x)$ defined by equality (2.9) and $\delta(h \beta)$ is the discrete delta function.

Then taking into account (4.7), (4.8) and theorems 4.1, 4.2, for optimal coefficients we have

$$
C_{\beta}=D_{m}(h \beta) * u(h \beta) .
$$

Thus if we will find the function $u(h \beta)$ then the optimal coefficients will be found from equality (4.13).

In order to calculate the convolution (4.13) it is required to find the representation of the function $u(h \beta)$ for all integer values of $\beta$. From equality (4.1) we get that $u(h \beta)=f_{m}(h \beta)$ when $h \beta \in[0,1]$. Now we need to find the representation of the function $u(h \beta)$ when $\beta<0$ and $\beta>N$. 
Since $C_{\beta}=0$ when $h \beta \notin[0,1]$ then

$$
C_{\beta}=D_{m}(h \beta) * u(h \beta)=0, \quad h \beta \notin[0,1] .
$$

Now we calculate the convolution $v(h \beta)=G(h \beta) * C_{\beta}$ when $h \beta \notin[0,1]$.

Suppose $\beta<0$ then taking into account equalities (2.9), (4.2), (4.3), (4.4), we have

$$
\begin{aligned}
& v(h \beta)=G(h \beta) * C_{\beta}=\sum_{\gamma=-\infty}^{\infty} C_{\gamma} G(h \beta-h \gamma)= \\
& =\sum_{\gamma=0}^{N} C_{\gamma} \frac{\operatorname{sign}(h \beta-h \gamma)}{2}\left(\frac{e^{h \beta-h \gamma}-e^{-h \beta+h \gamma}}{2}-\sum_{k=1}^{m-1} \frac{(h \beta-h \gamma)^{2 k-1}}{(2 k-1) !}\right)= \\
& =-\frac{1}{2} \sum_{\gamma=0}^{N} C_{\gamma}\left(\frac{e^{h \beta-h \gamma}-e^{-h \beta+h \gamma}}{2}-\sum_{k=1}^{m-1} \frac{(h \beta-h \gamma)^{2 k-1}}{(2 k-1) !}\right)= \\
& =-\frac{e^{h \beta}}{4} \sum_{\gamma=0}^{N} C_{\gamma} e^{-h \gamma}+\frac{e^{-h \beta}}{4} \sum_{\gamma=0}^{N} C_{\gamma} e^{h \gamma}+\frac{1}{2} \sum_{\gamma=0}^{N} C_{\gamma} \sum_{k=1}^{m-1} \frac{(h \beta-h \gamma)^{2 k-1}}{(2 k-1) !}= \\
& =-\frac{e^{h \beta}}{4}\left(1-e^{-1}\right)+\frac{e^{-h \beta}}{4} \sum_{\gamma=0}^{N} C_{\gamma} e^{h \gamma}+\frac{1}{2} \sum_{\gamma=0}^{N} C_{\gamma}\left(\sum_{k=1}^{\left[\frac{m+1}{2}\right]-1} \sum_{\alpha=0}^{2 k-1} \frac{(h \beta)^{2 k-1-\alpha}(-h \gamma)^{\alpha}}{(2 k-1-\alpha) ! \cdot \alpha !}+\right. \\
& \left.+\sum_{k=\left[\frac{m+1}{2}\right]}^{m-1} \sum_{\alpha=0}^{m-2} \frac{(h \beta)^{2 k-1-\alpha}(-h \gamma)^{\alpha}}{(2 k-1-\alpha) ! \cdot \alpha !}+\sum_{k=\left[\frac{m+1}{2}\right]}^{m-1} \sum_{\alpha=m-1}^{2 k-1} \frac{(h \beta)^{2 k-1-\alpha}(-h \gamma)^{\alpha}}{(2 k-1-\alpha) ! \cdot \alpha !}\right)= \\
& =-\frac{e^{h \beta}}{4}\left(1-e^{-1}\right)+\frac{e^{-h \beta}}{4} \sum_{\gamma=0}^{N} C_{\gamma} e^{h \gamma}+\frac{1}{2} \sum_{k=\left[\frac{m+1}{2}\right]}^{m-1} \sum_{\alpha=m-1}^{2 k-1} \frac{(h \beta)^{2 k-1-\alpha}(-1)^{\alpha}}{(2 k-1-\alpha) ! \cdot \alpha !} \sum_{\gamma=0}^{N} C_{\gamma}(h \gamma)^{\alpha}+ \\
& +\frac{1}{2}\left(\sum_{k=1}^{\left[\frac{m+1}{2}\right]-1} \sum_{\alpha=0}^{2 k-1} \frac{(h \beta)^{2 k-1-\alpha}(-1)^{\alpha}}{(2 k-1-\alpha) ! \cdot(\alpha+1) !}+\sum_{k=\left[\frac{m+1}{2}\right]}^{m-1} \sum_{\alpha=0}^{m-2} \frac{(h \beta)^{2 k-1-\alpha}(-1)^{\alpha}}{(2 k-1-\alpha) ! \cdot(\alpha+1) !}\right)= \\
& =-\frac{e^{h \beta}}{4}\left(1-e^{-1}\right)+D e^{-h \beta}+Q^{(2 m-3)}(h \beta)+Q_{m-2}(h \beta) .
\end{aligned}
$$

Thus we get

where

$$
v(h \beta)=-\frac{e^{h \beta}}{4}\left(1-e^{-1}\right)+D e^{-h \beta}+Q^{(2 m-3)}(h \beta)+Q_{m-2}(h \beta) .
$$

$$
\begin{aligned}
Q^{(2 m-3)}(h \beta) & =\frac{1}{2}\left(\sum_{k=1}^{\left[\frac{m+1}{2}\right]-1} \sum_{\alpha=0}^{2 k-1} \frac{(h \beta)^{2 k-1-\alpha}(-1)^{\alpha}}{(2 k-1-\alpha) ! \cdot(\alpha+1) !}+\right. \\
& \left.+\sum_{k=\left[\frac{m+1}{2}\right]}^{m-1} \sum_{\alpha=0}^{m-2} \frac{(h \beta)^{2 k-1-\alpha}(-1)^{\alpha}}{(2 k-1-\alpha) ! \cdot(\alpha+1) !}\right)
\end{aligned}
$$


is the polynomial of degree $2 m-3$ with respect to $(h \beta)$,

$$
Q_{m-2}(h \beta)=\frac{1}{2} \sum_{k=\left[\frac{m+1}{2}\right]}^{m-1} \sum_{\alpha=m-1}^{2 k-1} \frac{(h \beta)^{2 k-1-\alpha}(-1)^{\alpha}}{(2 k-1-\alpha) ! \cdot \alpha !} \sum_{\gamma=0}^{N} C_{\gamma}(h \gamma)^{\alpha}
$$

is unknown polynomial of degree $m-2$ with respect to $(h \beta)$,

$$
D=\frac{1}{4} \sum_{\gamma=0}^{N} C_{\gamma} e^{h \gamma}
$$

Similarly, in the case $\beta>N$ for the convolution $v(h \beta)=G(h \beta) * C_{\beta}$ we obtain

$$
v(h \beta)=\frac{e^{h \beta}}{4}\left(1-e^{-1}\right)-D e^{-h \beta}-Q^{(2 m-3)}(h \beta)-Q_{m-2}(h \beta) .
$$

We denote

$$
\begin{array}{ll}
Q_{m-2}^{(-)}(h \beta)=P_{m-2}(h \beta)+Q_{m-2}(h \beta), & a^{-}=d+D, \\
Q_{m-2}^{(+)}(h \beta)=P_{m-2}(h \beta)-Q_{m-2}(h \beta), & a^{+}=d-D .
\end{array}
$$

and taking into account (4.14), (4.18), (4.7) we get the following problem.

Problem B. Find the solution of the equation

$$
D_{m}(h \beta) * u(h \beta)=0, \quad h \beta \notin[0,1]
$$

having the form:

$$
u(h \beta)= \begin{cases}-\frac{e^{h \beta}}{4}\left(1-e^{-1}\right)+a^{-} e^{-h \beta}+Q^{(2 m-3)}(h \beta)+Q_{m-2}^{(-)}(h \beta), & \beta<0 \\ f_{m}(h \beta), & 0 \leq \beta \leq N \\ \frac{e^{h \beta}}{4}\left(1-e^{-1}\right)+a^{+} e^{-h \beta}-Q^{(2 m-3)}(h \beta)+Q_{m-2}^{(+)}(h \beta), & \beta>N .\end{cases}
$$

Here $Q_{m-2}^{(-)}(h \beta)$ and $Q_{m-2}^{(+)}(h \beta)$ are unknown polynomials of degree $m-2$ with respect to $h \beta, a^{-}$and $a^{+}$are unknown constants.

If we find $Q_{m-2}^{(-)}(h \beta), Q_{m-2}^{(+)}(h \beta), a^{-}$and $a^{+}$then from (4.19), (4.20) we have

$$
\begin{aligned}
P_{m-2}(h \beta) & =\frac{1}{2}\left(Q_{m-2}^{(-)}(h \beta)+Q_{m-2}^{(+)}(h \beta)\right), \quad d=\frac{1}{2}\left(a^{-}+a^{+}\right), \\
Q_{m-2}(h \beta) & =\frac{1}{2}\left(Q_{m-2}^{(-)}(h \beta)-Q_{m-2}^{(+)}(h \beta)\right), \quad D=\frac{1}{2}\left(a^{-}-a^{+}\right),
\end{aligned}
$$

Unknowns $Q_{m-2}^{(-)}(h \beta), Q_{m-2}^{(+)}(h \beta), a^{-}$and $a^{+}$can be found from the equation (4.21), using the function $D_{m}(h \beta)$. Then we can obtain explicit form of the function $u(h \beta)$ and find the optimal coefficients $C_{\beta}$. Thus, the problem $B$ and respectively the problem $A$ can be solved.

But here we will not find $Q_{m-2}^{(-)}(h \beta), Q_{m-2}^{(+)}(h \beta), a^{-}$and $a^{+}$. Instead of them, using $D_{m}(h \beta)$ and $u(h \beta)$, taking into account (4.13), we will find the expressions for optimal coefficients $C_{\beta}$ when $\beta=1, \ldots, N-1$.

We denote

$$
a_{k}=\frac{A_{k}}{\lambda_{k} p} \sum_{\gamma=1}^{\infty} \lambda_{k}^{\gamma}\left(-\frac{e^{-h \gamma}}{4}\left(1-e^{-1}\right)+Q^{(2 m-3)}(-h \gamma)+Q_{m-2}^{(-)}(-h \gamma)+a^{-} e^{h \gamma}-f_{m}(-h \gamma)\right) \text {, }
$$




$$
b_{k}=\frac{A_{k}}{\lambda_{k} p} \sum_{\gamma=1}^{\infty} \lambda_{k}^{\gamma}\left(\frac{e^{h \gamma+1}}{4}\left(1-e^{-1}\right)-Q^{(2 m-3)}(1+h \gamma)+Q_{m-2}^{(+)}(1+h \gamma)+a^{+} e^{-1-h \gamma}-f_{m}(1+h \gamma)\right) \text {, }
$$

Here $\lambda_{k}$ are the roots and $p$ is the leading coefficient of the polynomial $\mathcal{P}_{2 m-2}(\lambda)$ of degree $2 m-2$ defined by equality (4.12) and $\left|\lambda_{k}\right|<1$. The series in the notations (4.23), (4.24) are convergent.

The following is true

Theorem 4.3 (Theorem 3 of [23]). The coefficients of optimal quadrature formulas in the sense of Sard of the form (1.1) in the space $W_{2}^{(m, m-1)}(0,1)$ have the following form

$$
C_{\beta}=D_{m}(h \beta) * f_{m}(h \beta)+\sum_{k=1}^{m-1}\left(a_{k} \lambda_{k}^{\beta}+b_{k} \lambda_{k}^{N-\beta}\right), \quad \beta=1,2, \ldots, N-1,
$$

where $a_{k}$ and $b_{k}$ are unknowns and have the form (4.23) and (4.24) respectively, $\lambda_{k}$ are the roots of the polynomial $\mathcal{P}_{2 m-2}(\lambda)$ which defined by equality (4.12) and $\left|\lambda_{k}\right|<1$.

Proof. Suppose $\beta=\overline{1, N-1}$. Then from (4.13), using (4.9), (4.22) and theorem 4.2, we have

$$
\begin{gathered}
C_{\beta}=D_{m}(h \beta) * u(h \beta)=\sum_{\gamma=-\infty}^{\infty} D_{m}(h \beta-h \gamma) u(h \gamma)= \\
=\sum_{\gamma=-\infty}^{-1} D_{m}(h \beta-h \gamma) u(h \gamma)+\sum_{\gamma=0}^{N} D_{m}(h \beta-h \gamma) u(h \gamma)+\sum_{\gamma=N+1}^{\infty} D_{m}(h \beta-h \gamma) u(h \gamma) .
\end{gathered}
$$

Hence using the definition of convolution of discrete functions we get

$$
\begin{gathered}
C_{\beta}=D_{m}(h \beta) * f_{m}(h \beta)+ \\
+\sum_{\gamma=1}^{\infty} \sum_{k=1}^{m-1} \frac{A_{k}}{\lambda_{k} p} \lambda_{k}^{\beta+\gamma}\left(-\frac{e^{-h \gamma}}{4}\left(1-e^{-1}\right)+Q^{(2 m-3)}(-h \gamma)+Q_{m-2}^{(-)}(-h \gamma)+a^{-} e^{h \gamma}-f_{m}(-h \gamma)\right)+ \\
+\sum_{\gamma=1}^{\infty} \sum_{k=1}^{m-1} \frac{A_{k}}{\lambda_{k} p} \lambda_{k}^{N+\gamma-\beta}\left(\frac{e^{1+h \gamma}}{4}\left(1-e^{-1}\right)-Q^{(2 m-3)}(1+h \gamma)+Q_{m-2}^{(+)}(1+h \gamma)+a^{+} e^{-1-h \gamma}-\right. \\
\left.-f_{m}(1+h \gamma)\right) .
\end{gathered}
$$

Whence taking into account the notations (4.23), (4.24), we get (4.25).

Theorem 4.3 is proved.

From theorem 4.3 it is clear that in order to obtain explicit forms of the optimal coefficients $C_{\beta}$ in the space $W_{2}^{(m \cdot m-1)}(0,1)$ it is sufficient to find $a_{k}$ and $b_{k}(k=\overline{1, m-1})$. But here we will not to calculate series (4.23) and (4.24). Instead of them substituting the equality (4.25) into (4.1) we obtain identity with respect to $(h \beta)$. Whence, equating corresponding coefficients the left and the right hand sides of equation (4.1) we will find $a_{k}$ and $b_{k}$. And the coefficient $C_{0}$ and $C_{N}$ will be found from (4.3) when $\alpha=0$ and (4.4), respectively. Below we will do it.

It should be noted that the cases $m=1$ and $m=2$ are solved in the work [23] and the following theorems are proved. 
Theorem 4.4 (Theorem 4 of [23]). The coefficients of optimal quadrature formulas of the form (1.1) with equal spaced nodes in the space $W_{2}^{(1,0)}(0,1)$ are expressed by formulas

$$
C_{\beta}= \begin{cases}\frac{e^{h}-1}{e^{h}+1}, & \beta=0, N, \\ \frac{2\left(e^{h}-1\right)}{e^{h}+1}, & \beta=\overline{1, N-1,}\end{cases}
$$

where $h=1 / N, N=1,2, \ldots$

Theorem 4.5 (Theorem 5 of [23]). The coefficients of optimal quadrature formulas of the form (1.1) with equal spaced nodes in the space $W_{2}^{(2,1)}(0,1)$ are expressed by formulas

$$
C_{\beta}= \begin{cases}1-\frac{h}{e^{h}-1}-K(h)\left(\lambda_{1}-\lambda_{1}^{N}\right), & \beta=0, \\ h+K(h)\left(\left(e^{h}-\lambda_{1}\right) \lambda_{1}^{\beta}+\left(1-\lambda_{1} e^{h}\right) \lambda_{1}^{N-\beta}\right), & \beta=\overline{1, N-1,} \\ -1+\frac{e^{h} h}{e^{h}-1}-K(h)\left(\lambda_{1}-\lambda_{1}^{N}\right) e^{h}, & \beta=N,\end{cases}
$$

where

$$
\begin{gathered}
K(h)=\frac{\left(2 e^{h}-2-h e^{h}-h\right)\left(\lambda_{1}-1\right)}{2\left(e^{h}-1\right)^{2}\left(\lambda_{1}+\lambda_{1}^{N+1}\right)}, \\
\lambda_{1}=\frac{h\left(e^{2 h}+1\right)-e^{2 h}+1-\left(e^{h}-1\right) \sqrt{h^{2}\left(e^{h}+1\right)^{2}+2 h\left(1-e^{h}\right)}}{1-e^{2 h}+2 h e^{h}}, \quad\left|\lambda_{1}\right|<1,
\end{gathered}
$$

$h=1 / N, N=2,3, \ldots$

The main goal of the present section is to solve the system (4.1)-(4.4) for any $m \geq 2$ and any natural $N, N \geq m$. As mentioned above for this sufficiently to find $a_{k}$ and $b_{k}(k=\overline{1, m-1})$ in $(4.25)$.

The main result of the present paper is the following theorem.

Theorem 4.6. The coefficients of optimal quadrature formulas of the form (1.1) with the error functional (1.2) and with equal spaced nodes in the space $W_{2}^{(m, m-1)}(0,1)$ when $m \geq 2$ are expressed by formulas

$$
\begin{aligned}
& C_{0}=\frac{e^{h}-1-h}{e^{h}-1}+\sum_{k=1}^{m-1}\left(a_{k} \frac{\lambda_{k}\left(e^{h}-e\right)+\lambda_{k}^{2}(e-1)+\lambda_{k}^{N+1}\left(1-e^{h}\right)}{(e-1)\left(1-\lambda_{k}\right)\left(e^{h}-\lambda_{k}\right)}+b_{k} \frac{\lambda_{k}^{N+1}\left(e^{h}-e\right)+\lambda_{k}^{N}(e-1)+\lambda_{k}\left(1-e^{h}\right)}{(e-1)\left(\lambda_{k}-1\right)\left(\lambda_{k} e^{h}-1\right)}\right), \\
& C_{\beta}=h+\sum_{k=1}^{m-1}\left(a_{k} \lambda_{k}^{\beta}+b_{k} \lambda_{k}^{N-\beta}\right), \quad \beta=\overline{1, N-1}, \\
& C_{N}=\frac{e^{h} h+1-e^{h}}{e^{h}-1}+\sum_{k=1}^{m-1}\left(a_{k} \frac{\lambda_{k}\left(e-e^{h+1}\right)+\lambda_{k}^{N}\left(e^{h+1}-e^{h}\right)+\lambda_{k}^{N+1}\left(e^{h}-e\right)}{(e-1)\left(1-\lambda_{k}\right)\left(e^{h}-\lambda_{k}\right)}+b_{k} \frac{\lambda_{k}^{N+1}\left(e-e^{h+1}\right)+\lambda_{k}^{2}\left(e^{h+1}-e^{h}\right)+\lambda_{k}\left(e^{h}-e\right)}{(e-1)\left(1-\lambda_{k}\right)\left(1-\lambda_{k} e^{h}\right)}\right),
\end{aligned}
$$

where $a_{k}$ and $b_{k}(k=\overline{1, m-1})$ are defined by the following system of $2 m-2$ linear equations

$$
\begin{aligned}
& \sum_{k=1}^{m-1} a_{k} \frac{\lambda_{k}}{\left(\lambda_{k}-1\right)\left(\lambda_{k}-e^{h}\right)}+\sum_{k=1}^{m-1} b_{k} \frac{\lambda_{k}^{N+1}}{\left(\lambda_{k}-1\right)\left(\lambda_{k} e^{h}-1\right)}=\frac{h-2}{2\left(e^{h}-1\right)}+\frac{h}{\left(e^{h}-1\right)^{2}} ; \\
& \sum_{k=1}^{m-1} a_{k} \frac{\lambda_{k}^{N+1}}{\left(\lambda_{k}-1\right)\left(\lambda_{k}-e^{h}\right)}+\sum_{k=1}^{m-1} b_{k} \frac{\lambda_{k}}{\left(\lambda_{k}-1\right)\left(\lambda_{k} e^{h}-1\right)}=\frac{h-2}{2\left(e^{h}-1\right)}+\frac{h}{\left(e^{h}-1\right)^{2}} ; \\
& \sum_{k=1}^{m-1} a_{k}\left[\sum_{l=2}^{j} \frac{h^{2 l-2}}{(2 l-2) !} \sum_{i=1}^{2 l-2} \frac{\lambda_{k} \Delta^{i} 0^{2 l-2}}{\left(\lambda_{k}-1\right)^{i+1}}\right]+\sum_{k=1}^{m-1} b_{k}\left[\sum_{l=2}^{j} \frac{h^{2 l-2}}{(2 l-2) !} \sum_{i=1}^{2 l-2} \frac{\lambda_{k}^{N+i} \Delta^{i} 0^{2 l-2}}{\left(1-\lambda_{k}\right)^{i+1}}\right]=0, \quad j=\overline{2,\left[\frac{m}{2}\right] ;} \\
& \sum_{k=1}^{m-1} a_{k}\left[\sum_{l=1}^{j} \frac{h^{2 l-1}}{(2 l-1) !} \sum_{i=1}^{2 l-1} \frac{\lambda_{k} \Delta^{i} 0^{2 l-1}}{\left(\lambda_{k}-1\right)^{i+1}}\right]+\sum_{k=1}^{m-1} b_{k}\left[\sum_{l=1}^{j} \frac{h^{2 l-1}}{(2 l-1) !} \sum_{i=1}^{2 l-1} \frac{\lambda_{k}^{N+i} \Delta^{i} 0^{2 l-1}}{\left(1-\lambda_{k}\right)^{i+1}}\right]=\sum_{l=1}^{j} \frac{h^{2 l} B_{2 l} l}{(2 l) !}, \quad j=\overline{1,\left[\frac{m-1}{2}\right] ;}
\end{aligned}
$$




$$
\begin{aligned}
\sum_{k=1}^{m-1} a_{k} & {\left[\sum_{l=1}^{j} h^{l} C_{j}^{l} \sum_{i=1}^{l} \frac{\lambda_{k}^{N+i} \Delta^{i} 0^{l}}{\left(1-\lambda_{k}\right)^{i+1}}-h^{j} \sum_{i=1}^{j} \frac{\lambda_{k}^{i} \Delta^{i} 0^{j}}{\left(1-\lambda_{k}\right)^{i+1}}\right] } \\
& +\sum_{k=1}^{m-1} b_{k}\left[\sum_{l=1}^{j} h^{l} C_{j}^{l} \sum_{i=1}^{l} \frac{\lambda_{k} \Delta^{i} 0^{l}}{\left(\lambda_{k}-1\right)^{i+1}}-h^{j} \sum_{i=1}^{j} \frac{\lambda_{k}^{N+1} \Delta^{i} 0^{j}}{\left(\lambda_{k}-1\right)^{i+1}}\right]=\sum_{l=1}^{j-1} \frac{j ! B_{j+1-l}}{l !(j+1-l) !} h^{j+1-l}, \quad j=\overline{1, m-2 .}
\end{aligned}
$$

Here $\lambda_{k}$ are the roots of the polynomial (4.12) and $\left|\lambda_{k}\right|<1, B_{j}$ are Bernoulli numbers.

In the proof of theorem 4.6 we use the following formulas from [10]:

$$
\sum_{\gamma=0}^{n-1} q^{\gamma} \gamma^{k}=\frac{1}{1-q} \sum_{i=0}^{k}\left(\frac{q}{1-q}\right)^{i} \Delta^{i} 0^{k}-\left.\frac{q^{n}}{1-q} \sum_{i=0}^{k}\left(\frac{q}{1-q}\right)^{i} \Delta^{i} \gamma^{k}\right|_{\gamma=n},
$$

where $\Delta^{i} 0^{k}=\sum_{l=1}^{i}(-1)^{i-l} C_{i}^{l} l^{k}, \Delta^{i} \gamma^{k}$ is the finite difference of order $i$ of $\gamma^{k}$, and from $[9]$ :

$$
\sum_{\gamma=0}^{\beta-1} \gamma^{k}=\sum_{j=1}^{k+1} \frac{k ! B_{k+1-j}}{j !(k+1-j) !} \beta^{j}
$$

where $B_{k+1-j}$ are Bernoulli numbers,

$$
\Delta^{\alpha} x^{\nu}=\sum_{p=0}^{\nu}\left(\begin{array}{c}
\nu \\
p
\end{array}\right) \Delta^{\alpha} 0^{p} x^{\nu-p}
$$

Proof of theorem 4.6. For convenience we denote

$$
T=D_{m}(h \beta) * f_{m}(h \beta) .
$$

Now we consider equality (4.1)

$$
\sum_{\gamma=0}^{N} C_{\gamma} \frac{\operatorname{sign}(h \beta-h \gamma)}{2}\left(\frac{e^{h \beta-h \gamma}-e^{h \gamma-h \beta}}{2}-\sum_{k=1}^{m-1} \frac{(h \beta-h \gamma)^{2 k-1}}{(2 k-1) !}\right)+P_{m-2}(h \beta)+d e^{-h \beta}=f_{m}(h \beta),
$$

where $\beta=0,1, \ldots, N$.

We denote

$$
g(h \beta)=\sum_{\gamma=0}^{N} C_{\gamma} \frac{\operatorname{sign}(h \beta-h \gamma)}{2}\left(\frac{e^{h \beta-h \gamma}-e^{h \gamma-h \beta}}{2}-\sum_{k=1}^{m-1} \frac{(h \beta-h \gamma)^{2 k-1}}{(2 k-1) !}\right) .
$$

The expresson $g(h \beta)$ we rewrite in the following form

$$
\begin{aligned}
g(h \beta)= & C_{0}\left(\frac{e^{h \beta}-e^{-h \beta}}{2}-\sum_{k=1}^{m-1} \frac{(h \beta)^{2 k-1}}{(2 k-1) !}\right)+\sum_{\gamma=1}^{\beta-1} C_{\gamma}\left(\frac{e^{h \beta-h \gamma}-e^{h \gamma-h \beta}}{2}-\sum_{k=1}^{m-1} \frac{(h \beta-h \gamma)^{2 k-1}}{(2 k-1) !}\right) \\
& -\frac{1}{2} \sum_{\gamma=0}^{N} C_{\gamma}\left(\frac{e^{h \beta-h \gamma}-e^{h \gamma-h \beta}}{2}-\sum_{k=1}^{m-1} \frac{(h \beta-h \gamma)^{2 k-1}}{(2 k-1) !}\right) .
\end{aligned}
$$


Further we denote

$$
\begin{gathered}
g_{1}(h \beta)=\sum_{\gamma=0}^{\beta-1} C_{\gamma}\left(\frac{e^{h \beta-h \gamma}-e^{h \gamma-h \beta}}{2}-\sum_{k=1}^{m-1} \frac{(h \beta-h \gamma)^{2 k-1}}{(2 k-1) !}\right), \\
g_{2}(h \beta)=-\frac{1}{2} \sum_{\gamma=0}^{N} C_{\gamma}\left(\frac{e^{h \beta-h \gamma}-e^{h \gamma-h \beta}}{2}-\sum_{k=1}^{m-1} \frac{(h \beta-h \gamma)^{2 k-1}}{(2 k-1) !}\right) .
\end{gathered}
$$

Firstly we consider $g_{1}(h \beta)$ and rewrite (4.33) in the following form

$$
g_{1}(h \beta)=\sum_{\gamma=1}^{\beta-1} C_{\beta-\gamma}\left(\frac{e^{h \gamma}-e^{-h \gamma}}{2}-\sum_{k=1}^{m-1} \frac{(h \gamma)^{2 k-1}}{(2 k-1) !}\right) .
$$

Hence using (4.25), (4.29) we get

$$
\begin{gathered}
g_{1}(h \beta)=\sum_{\gamma=1}^{\beta-1}\left(T+\sum_{k=1}^{m-1}\left(a_{k} \lambda_{k}^{\beta-\gamma}+b_{k} \lambda_{k}^{N-\beta+\gamma}\right)\right) \frac{e^{h \gamma}}{2}- \\
-\sum_{\gamma=1}^{\beta-1}\left(T+\sum_{k=1}^{m-1}\left(a_{k} \lambda_{k}^{\beta-\gamma}+b_{k} \lambda_{k}^{N-\beta+\gamma}\right)\right) \frac{e^{-h \gamma}}{2}- \\
-\sum_{\ell=1}^{m-1} \sum_{\gamma=1}^{\beta-1}\left(T+\sum_{k=1}^{m-1}\left(a_{k} \lambda_{k}^{\beta-\gamma}+b_{k} \lambda_{k}^{N-\beta+\gamma}\right)\right) \frac{(h \gamma)^{2 \ell-1}}{(2 \ell-1) !} .
\end{gathered}
$$

From here taking into account (4.26), (4.27) and after some simplifications we have

$$
\begin{gathered}
g_{1}(h \beta)=\frac{T\left(e^{h}-e^{h \beta}\right)}{2\left(1-e^{h}\right)}+\sum_{k=1}^{m-1}\left(a_{k} \frac{\lambda_{k}^{\beta} e^{h}-\lambda_{k} e^{h \beta}}{2\left(\lambda_{k}-e^{h}\right)}+b_{k} \frac{\lambda_{k}^{N-\beta+1} e^{h}-\lambda_{k}^{N} e^{h \beta}}{2\left(1-\lambda_{k} e^{h}\right)}\right)- \\
-\frac{T\left(1-e^{h-h \beta}\right)}{2\left(e^{h}-1\right)}-\sum_{k=1}^{m-1}\left(a_{k} \frac{\lambda_{k}^{\beta}-\lambda_{k} e^{h-h \beta}}{2\left(\lambda_{k} e^{h}-1\right)}+b_{k} \frac{\lambda_{k}^{N-\beta+1}-\lambda_{k}^{N} e^{h-h \beta}}{2\left(e^{h}-\lambda_{k}\right)}\right)- \\
-\sum_{\ell=1}^{m-1} \frac{T h^{-1}(h \beta)^{2 l}}{(2 l) !}-\sum_{\ell=1}^{m-1} \sum_{j=1}^{2 \ell-1} \frac{T h^{2 \ell-1} B_{2 \ell-j}}{j !(2 \ell-j) !} \beta^{j}- \\
-\sum_{\ell=1}^{m-1} \frac{h^{2 \ell-1}}{(2 \ell-1) !} \sum_{k=1}^{m-1}\left[a_{k}\left(\frac{\lambda_{k}^{\beta+1}}{\lambda_{k}-1} \sum_{i=0}^{2 \ell-1} \frac{\Delta^{i} 0^{2 \ell-1}}{\left(\lambda_{k}-1\right)^{i}}-\frac{\lambda_{k}}{\lambda_{k}-1} \sum_{i=0}^{2 \ell-1} \frac{\Delta^{i} \beta^{2 \ell-1}}{\left(\lambda_{k}-1\right)^{i}}\right)+\right. \\
\left.+b_{k}\left(\frac{\lambda_{k}^{N-\beta}}{1-\lambda_{k}} \sum_{i=0}^{2 \ell-1}\left(\frac{\lambda_{k}}{1-\lambda_{k}}\right)^{i} \Delta^{i} 0^{2 \ell-1}-\frac{\lambda_{k}^{N}}{1-\lambda_{k}} \sum_{i=0}^{2 \ell-1}\left(\frac{\lambda_{k}}{1-\lambda_{k}}\right)^{i} \Delta^{i} \beta^{2 \ell-1}\right)\right] .
\end{gathered}
$$

In the last expression of $g_{1}(h \beta)$ the coefficients of $\lambda_{k}^{\beta}$ and $\lambda_{k}^{N-\beta}$ are the values of the polynomial $\mathcal{P}_{2 m-2}(\lambda)$ which defined by equality (4.12) at the $\lambda_{k}$. Since $\lambda_{k}$ are the roots of the polynomial (4.12), then the coefficients of $\lambda_{k}^{\beta}$ and $\lambda_{k}^{N-\beta}$ are zero. Then from (4.35) for $g_{1}(h \beta)$ we get

$$
g_{1}(h \beta)=\frac{e^{h \beta}}{2}\left[\frac{T}{e^{h}-1}+\sum_{k=1}^{m-1}\left(a_{k} \frac{\lambda_{k}}{e^{h}-\lambda_{k}}+b_{k} \frac{\lambda_{k}^{N}}{\lambda_{k} e^{h}-1}\right)\right]-
$$




$$
\begin{gathered}
-\frac{e^{-h \beta}}{2}\left[\frac{T e^{h}}{1-e^{h}}+\sum_{k=1}^{m-1}\left(a_{k} \frac{\lambda_{k} e^{h}}{1-e^{h} \lambda_{k}}+b_{k} \frac{\lambda_{k}^{N} e^{h}}{\lambda_{k}-e^{h}}\right)\right]+ \\
+\frac{T e^{h}}{2\left(1-e^{h}\right)}+\frac{T}{2\left(1-e^{h}\right)}-\sum_{\ell=1}^{m-1} \frac{T h^{-1}(h \beta)^{2 \ell}}{(2 \ell) !}-\sum_{\ell=1}^{m-1} T h^{2 \ell-1} \sum_{j=1}^{2 \ell-1} \frac{B_{2 \ell-j}}{j !(2 \ell-j) !} \beta^{j}+ \\
+\sum_{\ell=1}^{m-1} \frac{h^{2 \ell-1}}{(2 \ell-1) !} \sum_{k=1}^{m-1} a_{k} \frac{\lambda_{k}}{\lambda_{k}-1} \sum_{i=0}^{2 \ell-1} \frac{\Delta^{i} \beta^{2 \ell-1}}{\left(\lambda_{k}-1\right)^{i}}+\sum_{\ell=1}^{m-1} \frac{h^{2 \ell-1}}{(2 \ell-1) !} \sum_{k=1}^{m-1} b_{k} \frac{\lambda_{k}^{N}}{1-\lambda_{k}} \sum_{i=0}^{2 \ell-1}\left(\frac{\lambda_{k}}{1-\lambda_{k}}\right)^{i} \Delta^{i} \beta^{2 \ell-1} .
\end{gathered}
$$

Finally taking into account equality (4.28) we have

$$
\begin{gathered}
g_{1}(h \beta)=\frac{e^{h \beta}}{2}\left[\frac{T}{e^{h}-1}+\sum_{k=1}^{m-1}\left(a_{k} \frac{\lambda_{k}}{e^{h}-\lambda_{k}}+b_{k} \frac{\lambda_{k}^{N}}{\lambda_{k} e^{h}-1}\right)\right]- \\
-\frac{e^{-h \beta}}{2}\left[\frac{T e^{h}}{1-e^{h}}+\sum_{k=1}^{m-1}\left(a_{k} \frac{\lambda_{k} e^{h}}{1-e^{h} \lambda_{k}}+b_{k} \frac{\lambda_{k}^{N} e^{h}}{\lambda_{k}-e^{h}}\right)\right]+ \\
+\frac{T e^{h}}{2\left(1-e^{h}\right)}+\frac{T}{2\left(1-e^{h}\right)}-\sum_{\ell=1}^{m-1} \frac{T h^{-1}(h \beta)^{2 \ell}}{(2 \ell) !}-\sum_{\ell=1}^{m-1} T h^{2 \ell-1} \sum_{j=1}^{2 \ell-1} \frac{B_{2 \ell-j}}{j !(2 \ell-j) !} \beta^{j}+ \\
+\sum_{\ell=1}^{m-1} \frac{h^{2 \ell-1}}{(2 \ell-1) !} \sum_{j=0}^{2 \ell-1} C_{2 \ell-1}^{j} \beta^{j} \sum_{k=1}^{m-1} a_{k} \frac{\lambda_{k}}{\lambda_{k}-1} \sum_{i=0}^{2 \ell-1} \frac{\Delta^{i} 0^{2 \ell-1-j}}{\left(\lambda_{k}-1\right)^{i}}+ \\
+\sum_{\ell=1}^{m-1} \frac{h^{2 \ell-1}}{(2 \ell-1) !} \sum_{j=0}^{2 \ell-1} C_{2 \ell-1}^{j} \beta^{j} \sum_{k=1}^{m-1} b_{k} \frac{\lambda_{k}^{N}}{1-\lambda_{k}} \sum_{i=0}^{2 \ell-1}\left(\frac{\lambda_{k}}{1-\lambda_{k}}\right)^{i} \Delta^{i} 0^{2 \ell-1-j} .
\end{gathered}
$$

Now using the binomial formula and equalities (4.3), (4.4) from (4.34) we obtain

$$
\begin{array}{r}
g_{2}(h \beta)=-\frac{1}{2}\left(\frac{e^{h \beta}}{2} \sum_{\gamma=0}^{N} C_{\gamma} e^{-h \gamma}-\frac{e^{-h \beta}}{2} \sum_{\gamma=0}^{N} C_{\gamma} e^{h \gamma}-\sum_{\gamma=0}^{N} C_{\gamma} \sum_{k=1}^{m-1} \frac{(h \beta-h \gamma)^{2 m-1}}{(2 k-1) !}\right)= \\
=-\frac{1}{2}\left[\frac{e^{h \beta}}{2}\left(1-e^{-1}\right)-\frac{e^{-h \beta}}{2} \sum_{\gamma=0}^{N} C_{\gamma} e^{h \gamma}-\left(\sum_{k=1}^{\left[\frac{m+1}{2}\right]-1} \sum_{\alpha=0}^{2 k-1} \frac{(h \beta)^{2 k-1-\alpha}(-1)^{\alpha}}{(2 k-1-\alpha) !(\alpha+1) !}+\right.\right. \\
\left.\left.+\sum_{k=\left[\frac{m+1}{2}\right]}^{m-1} \sum_{\alpha=0}^{m-2} \frac{(h \beta)^{2 k-1-\alpha}(-1)^{\alpha}}{(2 k-1-\alpha) !(\alpha+1) !}+\sum_{k=\left[\frac{m+1}{2}\right]}^{m-1} \sum_{\alpha=m-1}^{2 k-1} \frac{(h \beta)^{2 k-1-\alpha}(-1)^{\alpha}}{(2 k-1-\alpha) ! \alpha !} \sum_{\gamma=0}^{N} C_{\gamma}(h \gamma)^{\alpha}\right)\right] .
\end{array}
$$

Using the binomial formula in equality (4.5) for $f_{m}(h \beta)$ we have

$$
\begin{aligned}
f_{m}(h \beta) & =\frac{e^{h \beta}+e^{-h \beta}+e^{1-h \beta}+e^{h \beta-1}-4}{4}-\sum_{k=1}^{m-1} \frac{(h \beta)^{2 k}}{(2 k) !}+\frac{1}{2} \sum_{k=1}^{\left[\frac{m+1}{2}\right]-1} \sum_{\alpha=0}^{2 k-1} \frac{(h \beta)^{2 k-1-\alpha}(-1)^{\alpha}}{(2 k-1-\alpha) !(\alpha+1) !}+ \\
+ & \frac{1}{2} \sum_{k=\left[\frac{m+1}{2}\right]}^{m-1} \sum_{\alpha=0}^{m-2} \frac{(h \beta)^{2 k-1-\alpha}(-1)^{\alpha}}{(2 k-1-\alpha) !(\alpha+1) !}+\frac{1}{2} \sum_{k=\left[\frac{m+1}{2}\right]} \sum_{\alpha=m-1}^{m-2} \frac{(h \beta)^{2 k-1-\alpha}(-1)^{\alpha}}{(2 k-1-\alpha) !(\alpha+1) !} .
\end{aligned}
$$


Taking into account (4.36), (4.37) and putting (4.32), (4.38) into (4.30) we get

$$
\begin{aligned}
& C_{0}\left[\frac{e^{h \beta}-e^{-h \beta}}{2}-\sum_{k=1}^{m-1} \frac{(h \beta)^{2 k-1}}{(2 k-1) !}\right]+\frac{e^{h \beta}}{2}\left[\frac{T}{e^{h}-1}+\sum_{k=1}^{m-1}\left(a_{k} \frac{\lambda_{k}}{e^{h}-\lambda_{k}}+b_{k} \frac{\lambda_{k}^{N}}{\lambda_{k} e^{h}-1}\right)\right]- \\
& -\frac{e^{-h \beta}}{2}\left[\frac{T e^{h}}{1-e^{h}}+\sum_{k=1}^{m-1}\left(a_{k} \frac{\lambda_{k} e^{h}}{1-e^{h} \lambda_{k}}+b_{k} \frac{\lambda_{k}^{N} e^{h}}{\lambda_{k}-e^{h}}\right)\right]+ \\
& +\frac{T e^{h}}{2\left(1-e^{h}\right)}+\frac{T}{2\left(1-e^{h}\right)}-\sum_{\ell=1}^{m-1} \frac{T h^{-1}(h \beta)^{2 \ell}}{(2 \ell) !}-\sum_{\ell=1}^{m-1} T h^{2 \ell-1} \sum_{j=1}^{2 \ell-1} \frac{B_{2 \ell-j}}{j !(2 \ell-j) !} \beta^{j}+ \\
& +\sum_{\ell=1}^{m-1} \frac{h^{2 \ell-1}}{(2 \ell-1) !} \sum_{j=0}^{2 \ell-1} C_{2 \ell-1}^{j} \beta^{j} \sum_{k=1}^{m-1} a_{k} \frac{\lambda_{k}}{\lambda_{k}-1} \sum_{i=0}^{2 \ell-1} \frac{\Delta^{i} 0^{2 \ell-1-j}}{\left(\lambda_{k}-1\right)^{i}}+ \\
& +\sum_{\ell=1}^{m-1} \frac{h^{2 \ell-1}}{(2 \ell-1) !} \sum_{j=0}^{2 \ell-1} C_{2 \ell-1}^{j} \beta^{j} \sum_{k=1}^{m-1} b_{k} \frac{\lambda_{k}^{N}}{1-\lambda_{k}} \sum_{i=0}^{2 \ell-1}\left(\frac{\lambda_{k}}{1-\lambda_{k}}\right)^{i} \Delta^{i} 0^{2 \ell-1-j}- \\
& -\frac{1}{2}\left[\frac{e^{h \beta}}{2}\left(1-e^{-1}\right)-\frac{e^{-h \beta}}{2} \sum_{\gamma=0}^{N} C_{\gamma} e^{h \gamma}-\left(\sum_{k=1}^{\left[\frac{m+1}{2}\right]-1} \sum_{\alpha=0}^{2 k-1} \frac{(h \beta)^{2 k-1-\alpha}(-1)^{\alpha}}{(2 k-1-\alpha) !(\alpha+1) !}+\right.\right. \\
& \left.\left.+\sum_{k=\left[\frac{m+1}{2}\right]}^{m-1} \sum_{\alpha=0}^{m-2} \frac{(h \beta)^{2 k-1-\alpha}(-1)^{\alpha}}{(2 k-1-\alpha) !(\alpha+1) !}+\sum_{k=\left[\frac{m+1}{2}\right]}^{m-1} \sum_{\alpha=m-1}^{2 k-1} \frac{(h \beta)^{2 k-1-\alpha}(-1)^{\alpha}}{(2 k-1-\alpha) ! \alpha !} \sum_{\gamma=0}^{N} C_{\gamma}(h \gamma)^{\alpha}\right)\right]+ \\
& +P_{m-2}(h \beta)+2 d \frac{e^{-h \beta}}{2}=\frac{e^{h \beta}+e^{-h \beta}+e^{1-h \beta}+e^{h \beta-1}-4}{4}-\sum_{k=1}^{m-1} \frac{(h \beta)^{2 k}}{(2 k) !}+ \\
& +\frac{1}{2} \sum_{k=1}^{\left[\frac{m+1}{2}\right]-1} \sum_{\alpha=0}^{2 k-1} \frac{(h \beta)^{2 k-1-\alpha}(-1)^{\alpha}}{(2 k-1-\alpha) !(\alpha+1) !}+\frac{1}{2} \sum_{k=\left[\frac{m+1}{2}\right]}^{m-1} \sum_{\alpha=0}^{m-2} \frac{(h \beta)^{2 k-1-\alpha}(-1)^{\alpha}}{(2 k-1-\alpha) !(\alpha+1) !}+ \\
& +\frac{1}{2} \sum_{k=\left[\frac{m+1}{2}\right]}^{m-1} \sum_{\alpha=m-1}^{m-2} \frac{(h \beta)^{2 k-1-\alpha}(-1)^{\alpha}}{(2 k-1-\alpha) !(\alpha+1) !} .
\end{aligned}
$$

Since the last equation is the identity with respect to $(h \beta)$ then from (4.39) equating the coefficients of the term $(h \beta)^{2 m-2}$ we obtain that for $m \geq 2$

$$
T=D_{m}(h \beta) * f_{m}(h \beta)=h .
$$

Note that equality (4.40) for some $m$ was proved by directly calculation of the convolution $D_{m}(h \beta) *$ $f_{m}(h \beta)$ in [23].

Taking into account (4.40) for optimal coefficients (4.25) the following formula holds

$$
C_{\beta}=h+\sum_{k=1}^{m-1}\left(a_{k} \lambda_{k}^{\beta}+b_{k} \lambda_{k}^{N-\beta}\right), \quad \beta=\overline{1, N-1} .
$$


Keeping in mind (4.40) after some simplifications from (4.39) we get

$$
\begin{aligned}
& \frac{e^{h \beta}}{2}\left[C_{0}+\frac{h}{e^{h}-1}+\sum_{k=1}^{m-1}\left(a_{k} \frac{\lambda_{k}}{e^{h}-\lambda_{k}}+b_{k} \frac{\lambda_{k}^{N}}{\lambda_{k} e^{h}-1}\right)-\frac{e-1}{2 e}\right]+ \\
&+\frac{e^{-h \beta}}{2}\left[-C_{0}-\frac{h e^{h}}{1-e^{h}}+\sum_{k=1}^{m-1}\left(a_{k} \frac{\lambda_{k} e^{h}}{1-e^{h} \lambda_{k}}+b_{k} \frac{\lambda_{k}^{N} e^{h}}{\lambda_{k}-e^{h}}\right)+\frac{1}{2} \sum_{\gamma=0}^{N} C_{\gamma} e^{h \gamma}+2 d\right]+ \\
&+\frac{h\left(1+e^{h}\right)}{2\left(1-e^{h}\right)}-C_{0} \sum_{k=1}^{m-1} \frac{(h \beta)^{2 k-1}}{(2 k-1) !}-\sum_{\ell=1}^{m-1} h^{2 \ell} \sum_{j=1}^{2 \ell-1} \frac{B_{2 \ell-j}}{j !(2 \ell-j) !} \beta^{j}+ \\
&+\sum_{\ell=1}^{m-1} \frac{h^{2 \ell-1}}{(2 \ell-1) !} \sum_{j=0}^{2 \ell-1} C_{2 \ell-1}^{j} \beta^{j} \sum_{k=1}^{m-1} a_{k} \frac{\lambda_{k}}{\lambda_{k}-1} \sum_{i=0}^{2 \ell-1} \frac{\Delta^{i} 0^{2 \ell-1-j}}{\left(\lambda_{k}-1\right)^{i}}+ \\
&+\sum_{\ell=1}^{m-1} \frac{h^{2 \ell-1}}{(2 \ell-1) !} \sum_{j=0}^{2 \ell-1} C_{2 \ell-1}^{j} \beta^{j} \sum_{k=1}^{m-1} b_{k} \frac{\lambda_{k}^{N}}{1-\lambda_{k}} \sum_{i=0}^{2 \ell-1}\left(\frac{\lambda_{k}}{1-\lambda_{k}}\right)^{i} \Delta^{i} 0^{2 \ell-1-j}+ \\
&+\frac{1}{2} \sum_{k=\left[\frac{m+1}{2}\right]}^{m-1} \sum_{\alpha=m-1}^{2 k-1} \frac{(h \beta)^{2 k-1-\alpha}(-1)^{\alpha}}{(2 k-1-\alpha) ! \alpha !} \sum_{\gamma=0}^{N} C_{\gamma}(h \gamma)^{\alpha}+P_{m-2}(h \beta)= \\
&=\frac{e^{h \beta}(1+e)}{4 e}+\frac{e^{-h \beta}(1+e)}{4}-1+\frac{1}{2} \sum_{k=\left[\frac{m+1}{2}\right]}^{m-1} \sum_{\alpha=m-1}^{2 k-1} \frac{(h \beta)^{2 k-1-\alpha}(-1)^{\alpha}}{(2 k-1-\alpha) !(\alpha+1) !} .
\end{aligned}
$$

As said above (4.42) is the identity with respect to $(h \beta)$. From (4.42) equating the corresponding coefficients of $e^{h \beta}, e^{-h \beta},(h \beta)^{\alpha}, \alpha=\overline{0,2 m-3}$ one can get the system of linear equations with respect to unknowns $a_{k}, b_{k}(k=\overline{1, m-1}), P_{m-2}(h \beta)$ and $d$.

Here we obtain the linear system for unknowns $a_{k}, b_{k}(k=\overline{1, m-1})$.

Equating the coefficients of $e^{h \beta}$ in the both sides of (4.41) we get the following equation

$$
\sum_{k=1}^{m-1}\left(a_{k} \frac{\lambda_{k}-\lambda_{k}^{N+1}}{\left(e^{h}-\lambda_{k}\right)\left(1-\lambda_{k}\right)}+b_{k} \frac{\lambda_{k}-\lambda_{k}^{N+1}}{\left(\lambda_{k} e^{h}-1\right)\left(1-\lambda_{k}\right)}\right)=0 .
$$

Further in (4.42) we consider the terms which consists of $(h \beta)^{\alpha}, \alpha=\overline{m-1,2 m-3}$ and we get the equation

$$
\begin{gathered}
\sum_{\ell=\left[\frac{m+1}{2}\right]}^{m-1}\left[-C_{0} \frac{(h \beta)^{2 \ell-1}}{(2 \ell-1) !}-\sum_{j=m-1}^{2 \ell-1}(h \beta)^{j} \frac{h^{2 \ell-j} B_{2 \ell-j}}{j !(2 \ell-j) !}+\sum_{j=m-1}^{2 \ell-1} \frac{(h \beta)^{j} h^{2 \ell-j-1}}{(2 \ell-1-j) ! j !} \sum_{k=1}^{m-1} a_{k} \sum_{i=0}^{2 \ell-1} \frac{\lambda_{k} \Delta^{i} 0^{2 \ell-1-j}}{\left(\lambda_{k}-1\right)^{i+1}}+\right. \\
\left.+\sum_{j=m-1}^{2 \ell-1}(h \beta)^{j} \frac{h^{2 \ell-j-1}}{(2 \ell-1-j) ! j !} \sum_{k=1}^{m-1} b_{k} \sum_{i=0}^{2 \ell-1} \frac{\lambda_{k}^{N+i} \Delta^{i} 0^{2 \ell-1-j}}{\left(1-\lambda_{k}\right)^{i+1}}\right]=0
\end{gathered}
$$

Now from equations (4.3) when $\alpha=0$ and (4.4) using identities (4.26), (4.27), (4.28) taking into account (4.41) after some simplifications for the coefficients $C_{0}$ and $C_{N}$ we get the following expressions which are asserted in the theorem: 


$$
\begin{gathered}
C_{0}=\frac{e^{h}-1-h}{e^{h}-1}+\sum_{k=1}^{m-1}\left(a_{k} \frac{\lambda_{k}\left(e^{h}-e\right)+\lambda_{k}^{2}(e-1)+\lambda_{k}^{N+1}\left(1-e^{h}\right)}{(e-1)\left(1-\lambda_{k}\right)\left(e^{h}-\lambda_{k}\right)}+\right. \\
\left.+b_{k} \frac{\lambda_{k}^{N+1}\left(e^{h}-e\right)+\lambda_{k}^{N}(e-1)+\lambda_{k}\left(1-e^{h}\right)}{(e-1)\left(\lambda_{k}-1\right)\left(\lambda_{k} e^{h}-1\right)}\right) \\
C_{N}=\frac{e^{h} h+1-e^{h}}{e^{h}-1}+\sum_{k=1}^{m-1}\left(a_{k} \frac{\lambda_{k}\left(e-e^{h+1}\right)+\lambda_{k}^{N}\left(e^{h+1}-e^{h}\right)+\lambda_{k}^{N+1}\left(e^{h}-e\right)}{(e-1)\left(1-\lambda_{k}\right)\left(e^{h}-\lambda_{k}\right)}+\right. \\
\left.+b_{k} \frac{\lambda_{k}^{N+1}\left(e-e^{h+1}\right)+\lambda_{k}^{2}\left(e^{h+1}-e^{h}\right)+\lambda_{k}\left(e^{h}-e\right)}{(e-1)\left(1-\lambda_{k}\right)\left(1-\lambda_{k} e^{h}\right)}\right) .
\end{gathered}
$$

From (4.44), using (4.45), grouping the coefficients of same degrees of $(h \beta)$ and equating to zero that coefficients for unknowns $a_{k}$ and $b_{k}$ we obtain the following $m-1$ linear equations

$$
\begin{aligned}
& \sum_{k=1}^{m-1} a_{k}\left[\sum_{l=1}^{j} \frac{h^{2 l-2}}{(2 l-2) !} \sum_{i=0}^{2 l-2} \frac{\lambda_{k} \Delta^{i} 0^{2 l-2}}{\left(\lambda_{k}-1\right)^{i+1}}-\frac{\lambda_{k}\left(e^{h}-e\right)+\lambda_{k}^{2}(e-1)+\lambda_{k}^{N+1}\left(1-e^{h}\right)}{(e-1)\left(\lambda_{k}-1\right)\left(\lambda_{k}-e^{h}\right)}\right]+ \\
& +\sum_{k=1}^{m-1} b_{k}\left[\sum_{l=1}^{j} \frac{h^{2 l-2}}{(2 l-2) !} \sum_{i=0}^{2 l-2} \frac{\lambda_{k}^{N+i} \Delta^{i} 0^{2 l-2}}{\left(1-\lambda_{k}\right)^{i+1}}-\frac{\lambda_{k}\left(1-e^{h}\right)+\lambda_{k}^{N}(e-1)+\lambda_{k}^{N+1}\left(e^{h}-e\right)}{(e-1)\left(\lambda_{k}-1\right)\left(\lambda_{k} e^{h}-1\right)}\right]=\frac{e^{h}-1-h}{e^{h}-1}-\frac{h}{2}, j=\overline{1,\left[\frac{m}{2}\right]} \\
& \sum_{k=1}^{m-1} a_{k}\left[\sum_{l=1}^{j} \frac{h^{2 l-1}}{(2 l-1) !} \sum_{i=0}^{2 l-1} \frac{\lambda_{k} \Delta^{i} 0^{2 l-1}}{\left(\lambda_{k}-1\right)^{i+1}}\right]+\sum_{k=1}^{m-1} b_{k}\left[\sum_{l=1}^{j} \frac{h^{2 l-1}}{(2 l-1) !} \sum_{i=0}^{2 l-1} \frac{\lambda_{k}^{N+i} \Delta^{i} 0^{2 l-1}}{\left(1-\lambda_{k}\right)^{i+1}}\right], j=\overline{1,\left[\frac{m-1}{2}\right]}
\end{aligned}
$$

Further from (4.3) when $\alpha=1, \ldots, m-2$ using identities (4.26), (4.27), (4.28) and the expression (4.46) for unknowns $a_{k}$ and $b_{k}$ we have $m-2$ linear equations

$$
\begin{aligned}
& \sum_{k=1}^{m-1} a_{k}\left[h^{j} \sum_{i=0}^{j} \frac{\lambda_{k}^{i}-\lambda_{k}^{N+i}}{\left(1-\lambda_{k}\right)^{i+1}} \Delta^{i} 0^{j}-\sum_{l=0}^{j-1} h^{l} C_{j}^{l} \sum_{i=0}^{l} \frac{\lambda_{k}^{N+i} \Delta^{i} 0^{l}}{\left(1-\lambda_{k}\right)^{i+1}}+\frac{\lambda_{k}\left(e-e^{h+1}\right)+\lambda_{k}^{N}\left(e^{h+1}-e^{h}\right)+\lambda_{k}^{N+1}\left(e^{h}-e\right)}{(e-1)\left(\lambda_{k}-1\right)\left(\lambda_{k}-e^{h}\right)}\right]+ \\
& +\sum_{k=1}^{m-1} b_{k}\left[h^{j} \sum_{i=0}^{j} \frac{\lambda_{k}^{N+1}-\lambda_{k}}{\left(\lambda_{k}-1\right)^{i+1}} \Delta^{i} 0^{j}-\sum_{l=0}^{j-1} h^{l} C_{j}^{l} \sum_{i=0}^{l} \frac{\lambda_{k} \Delta^{i} 0^{l}}{\left(\lambda_{k}-1\right)^{i+1}}+\frac{\lambda_{k}^{N+1}\left(e-e^{h+1}\right)+\lambda_{k}\left(e^{h}-e\right)+\lambda_{k}^{2}\left(e^{h+1}-e^{h}\right)}{(e-1)\left(\lambda_{k}-1\right)\left(\lambda_{k} e^{h}-1\right)}\right]= \\
& \quad=-\sum_{l=1}^{j} \frac{j ! B_{j+1-l}}{l !(j+1-l) !} h^{j+1-l}-\frac{e^{h} h+1-e^{h}}{e^{h}-1}, \quad j=\overline{1, m-2 .}
\end{aligned}
$$

After some simplifications system of equations (4.43), (4.47), (4.48), (4.49) we get the system which given in the assertion of the theorem.

Theorem 4.6 is proved.

From theorem 4.6 when $m=2$ we get theorem 4.5 .

For $m=3$ and $m=4$ from theorem 4.6 we we have the following results.

Corollary 4.1. The coefficients of optimal quadrature formulas of the form (1.1) with the error functional (1.2) and with equal spaced nodes in the space $W_{2}^{(3,2)}(0,1)$ are expressed by formulas $C_{0}=\frac{e^{h}-1-h}{e^{h}-1}+\sum_{k=1}^{2}\left(a_{k} \frac{\lambda_{k}\left(e^{h}-e\right)+\lambda_{k}^{2}(e-1)+\lambda_{k}^{N+1}\left(1-e^{h}\right)}{(e-1)\left(1-\lambda_{k}\right)\left(e^{h}-\lambda_{k}\right)}+b_{k} \frac{\lambda_{k}^{N+1}\left(e^{h}-e\right)+\lambda_{k}^{N}(e-1)+\lambda_{k}\left(1-e^{h}\right)}{(e-1)\left(\lambda_{k}-1\right)\left(\lambda_{k} e^{h}-1\right)}\right)$, 
$C_{\beta}=h+\sum_{k=1}^{2}\left(a_{k} \lambda_{k}^{\beta}+b_{k} \lambda_{k}^{N-\beta}\right), \quad \beta=\overline{1, N-1}$,

$C_{N}=\frac{e^{h} h+1-e^{h}}{e^{h}-1}+\sum_{k=1}^{2}\left(a_{k} \frac{\lambda_{k}\left(e-e^{h+1}\right)+\lambda_{k}^{N}\left(e^{h+1}-e^{h}\right)+\lambda_{k}^{N+1}\left(e^{h}-e\right)}{(e-1)\left(1-\lambda_{k}\right)\left(e^{h}-\lambda_{k}\right)}+b_{k} \frac{\lambda_{k}^{N+1}\left(e-e^{h+1}\right)+\lambda_{k}^{2}\left(e^{h+1}-e^{h}\right)+\lambda_{k}\left(e^{h}-e\right)}{(e-1)\left(1-\lambda_{k}\right)\left(1-\lambda_{k} e^{h}\right)}\right)$,

where $a_{k}$ and $b_{k}(k=\overline{1,2})$ are defined by the following system of linear equations

$$
\begin{aligned}
& \sum_{k=1}^{2} a_{k} \frac{\lambda_{k}}{\left(\lambda_{k}-1\right)\left(\lambda_{k}-e^{h}\right)}+\sum_{k=1}^{2} b_{k} \frac{\lambda_{k}^{N+1}}{\left(\lambda_{k}-1\right)\left(\lambda_{k} e^{h}-1\right)}=\frac{h-2}{2\left(e^{h}-1\right)}+\frac{h}{\left(e^{h}-1\right)^{2}} \\
& \sum_{k=1}^{2} a_{k} \frac{\lambda_{k}^{N+1}}{\left(\lambda_{k}-1\right)\left(\lambda_{k}-e^{h}\right)}+\sum_{k=1}^{2} b_{k} \frac{\lambda_{k}}{\left(\lambda_{k}-1\right)\left(\lambda_{k} e^{h}-1\right)}=\frac{h-2}{2\left(e^{h}-1\right)}+\frac{h}{\left(e^{h}-1\right)^{2}} \\
& \sum_{k=1}^{2} a_{k} \frac{\lambda_{k}}{\left(\lambda_{k}-1\right)^{2}}+\sum_{k=1}^{2} b_{k} \frac{\lambda_{k}^{N+1}}{\left(\lambda_{k}-1\right)^{2}}=\frac{h}{12} \\
& \sum_{k=1}^{2} a_{k} \frac{\lambda_{k}^{N+1}}{\left(\lambda_{k}-1\right)^{2}}+\sum_{k=1}^{2} b_{k} \frac{\lambda_{k}}{\left(\lambda_{k}-1\right)^{2}}=\frac{h}{12}
\end{aligned}
$$

here $\lambda_{k}, k=1,2$ are the roots of the polynomial

$$
\mathcal{P}_{4}(\lambda)=\left(1-e^{2 h}\right)(1-\lambda)^{4}-2\left(\lambda\left(e^{2 h}+1\right)-e^{h}\left(\lambda^{2}+1\right)\right)\left(h(1-\lambda)^{2}+\frac{h^{3}}{6}\left(1+4 \lambda+\lambda^{2}\right)\right)
$$

which $\left|\lambda_{k}\right|<1$.

Corollary 4.2. The coefficients of optimal quadrature formulas of the form (1.1) with the error functional (1.2) and with equal spaced nodes in the space $W_{2}^{(4,3)}(0,1)$ are expressed by formulas $C_{0}=\frac{e^{h}-1-h}{e^{h}-1}+\sum_{k=1}^{3}\left(a_{k} \frac{\lambda_{k}\left(e^{h}-e\right)+\lambda_{k}^{2}(e-1)+\lambda_{k}^{N+1}\left(1-e^{h}\right)}{(e-1)\left(1-\lambda_{k}\right)\left(e^{h}-\lambda_{k}\right)}+b_{k} \frac{\lambda_{k}^{N+1}\left(e^{h}-e\right)+\lambda_{k}^{N}(e-1)+\lambda_{k}\left(1-e^{h}\right)}{(e-1)\left(\lambda_{k}-1\right)\left(\lambda_{k} e^{h}-1\right)}\right)$, $C_{\beta}=h+\sum_{k=1}^{3}\left(a_{k} \lambda_{k}^{\beta}+b_{k} \lambda_{k}^{N-\beta}\right), \quad \beta=\overline{1, N-1}$

$C_{N}=\frac{e^{h} h+1-e^{h}}{e^{h}-1}+\sum_{k=1}^{3}\left(a_{k} \frac{\lambda_{k}\left(e-e^{h+1}\right)+\lambda_{k}^{N}\left(e^{h+1}-e^{h}\right)+\lambda_{k}^{N+1}\left(e^{h}-e\right)}{(e-1)\left(1-\lambda_{k}\right)\left(e^{h}-\lambda_{k}\right)}+b_{k} \frac{\lambda_{k}^{N+1}\left(e-e^{h+1}\right)+\lambda_{k}^{2}\left(e^{h+1}-e^{h}\right)+\lambda_{k}\left(e^{h}-e\right)}{(e-1)\left(1-\lambda_{k}\right)\left(1-\lambda_{k} e^{h}\right)}\right)$, where $a_{k}$ and $b_{k}(k=\overline{1,3})$ are defined by the following system of linear equations

$$
\begin{aligned}
& \sum_{k=1}^{3} a_{k} \frac{\lambda_{k}}{\left(\lambda_{k}-1\right)\left(\lambda_{k}-e^{h}\right)}+\sum_{k=1}^{3} b_{k} \frac{\lambda_{k}^{N+1}}{\left(\lambda_{k}-1\right)\left(\lambda_{k} e^{h}-1\right)}=\frac{h-2}{2\left(e^{h}-1\right)}+\frac{h}{\left(e^{h}-1\right)^{2}} \\
& \sum_{k=1}^{3} a_{k} \frac{\lambda_{k}^{N+1}}{\left(\lambda_{k}-1\right)\left(\lambda_{k}-e^{h}\right)}+\sum_{k=1}^{3} b_{k} \frac{\lambda_{k}}{\left(\lambda_{k}-1\right)\left(\lambda_{k} e^{h}-1\right)}=\frac{h-2}{2\left(e^{h}-1\right)}+\frac{h}{\left(e^{h}-1\right)^{2}} \\
& \sum_{k=1}^{3} a_{k} \frac{\lambda_{k}}{\left(\lambda_{k}-1\right)^{2}}+\sum_{k=1}^{3} b_{k} \frac{\lambda_{k}^{N+1}}{\left(\lambda_{k}-1\right)^{2}}=\frac{h}{12} \\
& \sum_{k=1}^{3} a_{k} \frac{\lambda_{k}^{N+1}}{\left(\lambda_{k}-1\right)^{2}}+\sum_{k=1}^{3} b_{k} \frac{\lambda_{k}}{\left(\lambda_{k}-1\right)^{2}}=\frac{h}{12} \\
& \sum_{k=1}^{3} a_{k} \frac{\lambda_{k}}{\left(\lambda_{k}-1\right)^{3}}+\sum_{k=1}^{3} b_{k} \frac{\lambda_{k}^{N+2}}{\left(1-\lambda_{k}\right)^{3}}=-\frac{h}{24} \\
& \sum_{k=1}^{3} a_{k} \frac{\lambda_{k}^{2}-\lambda_{k}^{N+2}}{\left(1-\lambda_{k}\right)^{3}}+\sum_{k=1}^{3} b_{k} \frac{\lambda_{k}^{N+1}-\lambda_{k}}{\left(\lambda_{k}-1\right)^{3}}=0
\end{aligned}
$$


here $\lambda_{k}, k=1,2,3$ are the roots of the polynomial

$$
\begin{aligned}
\mathcal{P}_{6}(\lambda)= & \left(1-e^{2 h}\right)(1-\lambda)^{6}-2\left(\lambda\left(e^{2 h}+1\right)-e^{h}\left(\lambda^{2}+1\right)\right) \times \\
& \times\left(h(1-\lambda)^{4}+\frac{h^{3}}{6}(1-\lambda)^{2}\left(1+4 \lambda+\lambda^{2}\right)+\frac{h^{5}}{120}\left(1+26 \lambda+66 \lambda^{2}+26 \lambda^{3}+\lambda^{4}\right)\right)
\end{aligned}
$$

which $\left|\lambda_{k}\right|<1$.

\section{Numerical RESUlts}

In this section we give the numerical results which confirm the theoretical results obtained in the section 4 .

Taking into account (2.9) from (2.21) for the norm of the error functional of optimal quadrature formulas in the space $W_{2}^{(m, m-1)}(0,1)$ we obtain

$$
\begin{aligned}
\|\ell(x)\|^{2}= & (-1)^{m}\left[\sum_{\beta=0}^{N} \sum_{\gamma=0}^{N} C_{\beta} C_{\gamma} \frac{\operatorname{sing}(h \beta-h \gamma)}{2}\left(\frac{e^{h \beta-h \gamma}-e^{h \gamma-h \beta}}{2}-\sum_{k=1}^{m-1} \frac{(h \beta-h \gamma)^{2 k-1}}{(2 k-1) !}\right)-\right. \\
& -2 \sum_{\beta=0}^{N} C_{\beta}\left(\frac{e^{h \beta}+e^{h \beta}+e^{1-h \beta}+e^{h \beta-1}-4}{4}-\sum_{k=1}^{m-1} \frac{(h \beta)^{2 k}+(1-h \beta)^{2 k}}{2 \cdot(2 k) !}\right)+ \\
& \left.+\frac{e^{2}-2 e-1}{2 e}-\sum_{k=1}^{m-1} \frac{1}{(2 k+1) !}\right],
\end{aligned}
$$

In computations of optimal coefficients we need the roots $\lambda_{k}, k=\overline{1, m-1},\left|\lambda_{k}\right|<1$ of the polynomial defined by equality (4.12)

$$
\begin{aligned}
\mathcal{P}_{2 m-2}(\lambda) & =\sum_{s=0}^{2 m-2} p_{s}^{(2 m-2)} \lambda^{s}=\left(1-e^{2 h}\right)(1-\lambda)^{2 m-2}-2\left(\lambda\left(e^{2 h}+1\right)-e^{h}\left(\lambda^{2}+1\right)\right) \times \\
\times & {\left[h(1-\lambda)^{2 m-4}+\frac{h^{3}(1-\lambda)^{2 m-6}}{3 !} E_{2}(\lambda)+\ldots+\frac{h^{2 m-3} E_{2 m-4}(\lambda)}{(2 m-3) !}\right], }
\end{aligned}
$$

where $E_{2 k}(\lambda)=\sum_{s=0}^{2 k} e_{s} \lambda^{s}$ is the Euler-Frobenius polynomial of degree $2 k$ and for the coefficients of this polynomial the formula $e_{s}=\sum_{j=0}^{s}(-1)^{j}\left(\begin{array}{c}2 k+2 \\ j\end{array}\right)(s+1-j)^{2 k+1}$ is used which was given by Euler.

For convenience the absolute value of the difference (1.4) of the quadrature formula (1.1) we denote by $|R(\varphi)|$. Then by Cauchy-Scwartz inequality we have

$$
|R(\varphi)| \leq\left\|\varphi(x)\left|W_{2}^{(m, m-1)}(0,1)\|\cdot\| \ell(x)\right| W_{2}^{(m, m-1) *}(0,1)\right\| .
$$

We consider the cases $m=1,2,3,4$ and $N=10,50,100$.

The case $m=1$. In the space $W_{2}^{(1,0)}(0,1)$ using theorem 4.4 and (5.1), (5.3) for the error of optimal quadrature formula (1.1) we have

$$
\begin{array}{lll}
N=10: & & |R(\varphi)| \leq\left\|\varphi(x) \mid W_{2}^{(1,0)}(0,1)\right\| \cdot 0.02886 \\
N=50: & & |R(\varphi)| \leq\left\|\varphi(x) \mid W_{2}^{(1,0)}(0,1)\right\| \cdot 0.00577 \\
N=100: & & |R(\varphi)| \leq\left\|\varphi(x) \mid W_{2}^{(1,0)}(0,1)\right\| \cdot 0.00289 .
\end{array}
$$


The case $m=2$. In the space $W_{2}^{(2,1)}(0,1)$ using theorem 4.5 and (5.1), (5.2), (5.3) for the error of optimal quadrature formula (1.1) we have

$$
\begin{array}{lll}
N=10: & & |R(\varphi)| \leq\left\|\varphi(x) \mid W_{2}^{(2,1)}(0,1)\right\| \cdot 0.000424 \\
N=50: & & |R(\varphi)| \leq\left\|\varphi(x) \mid W_{2}^{(2,1)}(0,1)\right\| \cdot 0.00001534 \\
N=100: & & |R(\varphi)| \leq\left\|\varphi(x) \mid W_{2}^{(2,1)}(0,1)\right\| \cdot 0.37802 \times 10^{-5}
\end{array}
$$

The case $m=3$. In the space $W_{2}^{(3,2)}(0,1)$ using corollary 4.1 and (5.1), (5.2), (5.3) for the error of optimal quadrature formula (1.1) we have

$$
\begin{array}{lll}
N=10: & & |R(\varphi)| \leq\left\|\varphi(x) \mid W_{2}^{(3,2)}(0,1)\right\| \cdot 0.0000108 ; \\
N=50: & & |R(\varphi)| \leq\left\|\varphi(x) \mid W_{2}^{(3,2)}(0,1)\right\| \cdot 0.5643 \times 10^{-7} \\
N=100: & & |R(\varphi)| \leq\left\|\varphi(x) \mid W_{2}^{(3,2)}(0,1)\right\| \cdot 0.6435 \times 10^{-8}
\end{array}
$$

The case $m=4$. In the space $W_{2}^{(4,3)}(0,1)$ using corollary 4.2 and (5.1), (5.2), (5.3) for the error of optimal quadrature formula (1.1) we have

$$
\begin{array}{lll}
N=10: & |R(\varphi)| \leq\left\|\varphi(x) \mid W_{2}^{(4,3)}(0,1)\right\| \cdot 0.5051 \times 10^{-6} \\
N=50: & & |R(\varphi)| \leq\left\|\varphi(x) \mid W_{2}^{(4,3)}(0,1)\right\| \cdot 0.3854 \times 10^{-9} \\
N=100: & & |R(\varphi)| \leq\left\|\varphi(x) \mid W_{2}^{(4,3)}(0,1)\right\| \cdot 0.1821 \times 10^{-10}
\end{array}
$$

\section{ACKNOWLEDGEMENTS}

The authors are very thankful to professor Erich Novak for discussion of the results of this paper.

The final part of this work was done in the Friedrich-Schiller University of Jena, Germany. The second author thanks the DAAD for scholarship. Furthermore the second author also thanks IMU/CDEprogram for travel support to the Friedrich-Schiller University of Jena, Germany.

\section{REFERENCES}

[1] Ivo Babuška. Optimal quadrature formulas. Dokladi Akadem. Nauk SSSR. 1963. Vol.149. No.2. pp. 227-229. (in Russian)

[2] P.Blaga and Gh.Coman. Some problems on optimal quadrature. Studia Univ. "Babeş-Bolyai", Methematica, Vol.LII, No.4, December 2007. pp.21-44.

[3] B.Bojanov. Optimal quadrature formulas. Uspekhi Matemat. nauk. V.60, 6(366), 2005, pp. 33-52. (in Russian)

[4] T.Catinaş and Gh.Coman. Optimal quadrature formulas based on the $\varphi$-function method. Studia Univ. "BabeşBolyai", Mathematica, Vol.LII, No.6, January 2005. pp. 1-16.

[5] M.A.Chakhkiev. Linear differential operators with real spectrum and optimal quadrature formulas. Izvestiya of AS of USSR, V48, No5, 1984, pp. 1078-1108. (in Russian)

[6] Gh.Coman. Formule de cuadrature de tip Sard. Studia Univ. "Babeş- Bolyai". Ser. math.-mech., 17. No.2. - 1972. - pp. 73-77.,

[7] Gh.Coman. Monosplines and optimal quadrature Formule in $L_{p}$. Rendiconti di Matematica. 5. (1972). - No.3. -pp. 567-577.

[8] A.Ghizzetti, A.Ossicini. Quadrature Formulae, Berlin. Akademie Verlag, 1970.

[9] A.O.Gelfond, Calculus of Finite Differences, Nauka, Moscow, 1967, 376 p. (in Russian)

[10] R.W.Hamming, Numerical Methods for Scientists and Engineers, McGraw Bill Book Company, Inc., USA, 1962, $411 \mathrm{p}$.

[11] P.Köhler. On the Weights of Sard's Quadrature Formulas. Calcolo 25 (3), (1988), 169-186.

[12] F.Lanzara. On Optimal Quadrature Formulae. J. of Inequality \& Appl., 2000, Vol.5, pp.201-225.

[13] A.A.Malukov, I.I.Orlov. Construction of the coefficients of the best quadrature formula for class of equal spaced nodes. Applied mathematics. -Irkutsk, 1976. -P. 174-177. (in Russian) 
[14] L.F.Meyers, A.Sard. Best approximate integration formulas. // J. Math and Phys. - 1950. XXIX. pp. 118-123.

[15] S.M.Nikolskii. To question about estimation of approximation by quadrature formulas.-Uspekhi Matem. Nauk, 1950, $5: 2$ (36), pp. 165-177. (in Russian)

[16] S.M.Nikolskii. Quadrature formulas. -M.: Nauka, 1988 (in Russian)

[17] A.Sard. Best approximate integration formulas, best approximate formulas. // American J. of Math. 1949. LXXI. pp. 80-91.

[18] Kh.M.Shadimetov. Optimal quadrature formulas in the $L_{2}^{(m)}(\Omega)$ and $L_{2}^{(m)}\left(R^{1}\right)$, Dokl. Akademii Nauk UzSSR, No3, (1983), pp.5-8. (in Russian)

[19] Kh.M.Shadimetov. Construction of weight optimal quadrature formulas in the space $L_{2}^{(m)}(0, N)$, Siberian Journal of Computational Mathematics, V.5, No3, (2002), pp.275-293. (in Russian)

[20] Kh.M.Shadimetov, A.R.Hayotov. Optimal quadrature formulas with positive coefficients in $L_{2}^{(m)}(0,1)$ space, Journal of Computational and Applied Mathematics (2010), doi: 10.1016/j.cam.2010.07.021.

[21] Kh.M.Shadimetov, A.R.Hayotov. Construction of the discrete analogue of the differential operator $d^{2 m} / d x^{2 m}-$ $d^{2 m-2} / d x^{2 m-2}$. Uzbek Mathematical journal, 2004, No2, pp.85-95.

[22] Kh.M.Shadimetov, A.R.Hayotov. Properties of the discrete analogue of the differential operator $d^{2 m} / d x^{2 m}-$ $d^{2 m-2} / d x^{2 m-2}$. Uzbek Mathematical Journal. 2004, No.4. p. 72-83. (ArXiv.0810.5423v1 [math.NA])

[23] Kh.M.Shadimetov, A.R.Hayotov. Computation the coefficients of optimal quadrature formulas in the space $W_{2}^{(m, m-1)}(0,1)$. Uzbek Math. Journal. 2004, No3. p. 67-82. (arXiv:0810.5421v1 [math.NA])

[24] I.J.Schoenberg. On Monosplines of Least Deviation and Best Quadrature Formulae. Journal of the Society for Industrial and Applied Mathematics: Series B. Numerical Analysis, Vol.2, No.1, (1965), pp.144-170.

[25] I.J.Schoenberg. On Monosplines of Least Square Deviation and Best Quadrature Formulae II. SIAM Journal on Numerical Analysis, Vol.3, No.2, (1966), pp.321-328.

[26] I.J.Schoenberg and S.D.Silliman. On semicardinal quadrature formulae. Math. Comp., 1974. v. 126. pp. $483-497$.

[27] S.L.Sobolev. The coefficients of optimal quadrature formulas, in Selected Works of S.L.Sobolev. Springer, (2006). pp.561-566.

[28] S.L.Sobolev. Introduction to the theory of cubature formulas. -M.:Nauka, 1974, 808 p. (in Russian)

[29] S.L.Sobolev, V.L.Vaskevich. The Theory of Cubature Formulas, Kluwer Academic Publishers Group, Dordrecht, (1997). $416 \mathrm{p}$.

[30] V.S.Vladimirov. Generalized functions in mathematical physics .-M.: Nauka, 1979. 320 p. (in Russian)

[31] F.Ya.Zagirova. On construction of optimal quadrature formulas with equal spaced nodes.- Novosibirsk, (1982), 28 p. (Preprint No 25, Institute of Mathematics SD of AS of USSR). (in Russian)

[32] Z.Zh.Zhamalov, Kh.M.Shadimetov. About optimal quadrature formulas. Dokl. Akademii Nauk UzSSR, No7, (1980), pp.3-5. (in Russian)

[33] A.A.Zhensikbaev. Monosplines of minimal norm and the best quadrature formulas. - Uspekhi Matem. nauk. Vol.36, 4, 1981, pp. 107-159. (in Russian)

Kh. M. Shadimetov, A. R. Hayotov, Institute of mathematics and information technologies, Tashkent, UZBEKISTAN.

E-mail address: hayotov@mail.ru 